\title{
Jeans modeling of axisymmetric galaxies with multiple stellar populations
}

\author{
Caterina Caravita, ${ }^{1,2}{ }^{\star}$ Luca Ciotti $^{1}$ and Silvia Pellegrini ${ }^{1,2}$ \\ ${ }^{1}$ Department of Physics and Astronomy, University of Bologna, via P. Gobetti 93/2, 40129 Bologna, Italy \\ ${ }^{2}$ INAF-OAS of Bologna, via P. Gobetti 93/3, 40129 Bologna, Italy
}

22 June 2021

\begin{abstract}
We present the theoretical framework to efficiently solve the Jeans equations for multi-component axisymmetric stellar systems, focusing on the scaling of all quantities entering them. The models may include an arbitrary number of stellar distributions, a dark matter halo, and a central supermassive black hole; each stellar distribution is implicitly described by a two- or three-integral distribution function, and the stellar components can have different structural (density profile, flattening, mass, scale-length), dynamical (rotation, velocity dispersion anisotropy), and population (age, metallicity, initial mass function, mass-to-light ratio) properties. In order to determine the ordered rotational velocity and the azimuthal velocity dispersion fields of each component, we introduce a decomposition that can be used when the commonly adopted Satoh decomposition cannot be applied. The scheme developed is particularly suitable for a numerical implementation; we describe its realisation within our code JASMINE2, optimised to maximally exploit the scalings allowed by the Poisson and the Jeans equations, also in the post-processing procedures. As applications, we illustrate the building of three multi-component galaxy models with two distinct stellar populations, a central black hole, and a dark matter halo; we also study the solution of the Jeans equations for an exponential thick disc, and for its multi-component representation as the superposition of three Miyamoto-Nagai discs. A useful general formula for the numerical evaluation of the gravitational potential of factorised thick discs is finally given.
\end{abstract}

Key words: galaxies: structure - galaxies: kinematics and dynamics - methods: analytical - methods: numerical

\section{INTRODUCTION}

Axisymmetric galaxy models often represent an acceptable description of real galaxies, beyond the zeroth-order approximation of spherical symmetry. Analytical models of one and multi-component axisymmetric galaxies are available (e.g., see Binney \& Tremaine 2008, hereafter BT08, and references therein; see also Ciotti et al. 2021, hereafter CMPZ21), but these models, while important to highlight fundamental properties of the dynamics of axisymmetric systems, and to guide the construction of realistic galaxy models to be carried out numerically, suffer from the restrictions imposed by the request of analytical tractability. From this point of view, analytical and numerical modeling should be seen as complementary approaches, each of them with their own merits and limitations.

On the numerical side, the most common models are based on the solution of the Jeans equations (e.g. BT08; Ciotti 2021). This approach allows to model axisymmetric stellar systems, in the simplest assumption of a 2-integral phase-space distribution function (DF, e.g. Posacki et al. 2013), or a 3-integral DF (e.g. Cappellari 2008), starting from the assignment of the density components. As well known, the proper description of a stellar system should start from the assignment of the phase-space DF of each separate mass component, and the solution of the associated Poisson equation (BT08; Bertin 2014; Ciotti 2021); however, in several applications, the Jeans approach is highly preferred, for its direct control on the density

\footnotetext{
^ E-mail: caterina.caravita2@unibo.it
}

distributions (even if it leaves open fundamental issues such as the phase-space consistency).

In this paper we present a procedure especially designed to build (and project) multi-component systems. For an arbitrary number of mass components, we start with maximally exploiting the scalings allowed by the Poisson and the Jeans equations, and then we show how to combine the solutions for all components to obtain a particular multi-component model. When implemented numerically, this scheme allows for a fast and flexible building of realistic models. To provide an example, we describe how the various steps of the procedure were inserted in our code JASMINE (Jeans AxiSymmetric Models of galaxies IN Equilibrium, Posacki et al. 2013) for the axisymmetric modeling of galaxies based on the Jeans equations; the resulting much extended code version was named JASMINE2. This new code allows for the choice, in input, of different stellar components and Dark Matter (DM) components (from a continuously updated library), and a central Black Hole (BH). It computes numerically the gravitational potential of each density component by using the well-known formula based on complete elliptic integrals of the first kind. This numerical method is highly accurate, but it easily becomes quite time expensive, depending on the grid resolution and on the number of density components; if one wants to explore the parameter space (that can be very large, especially for multi-component models), the possibility of a scheme to allow for a full scaling of the Poisson and the Jeans equations is crucial. The building of a model is then organised in two distinct parts: in the first one, whose numerical realisation we call Potential and Jeans Solver, one computes the 
potential and then solves the Jeans equations for each scaled stellar density component; this produces a set of solutions that represents a "progenitor" of a family of models. In the second part, that in our numerical realisation is seen as a Post-Processing (PP) phase, the mass and luminosity weights are assigned, and the kinematical decompositions imposed; with these, the scaled solutions of the progenitor are finally combined, and the resulting kinematical fields projected.

This procedure allows to drastically reduce the computational time needed for the construction of a multi-component model: with a single run of the Potential and Jeans Solver, one can build a family of galaxy models, all characterised by the same set of scaled density components; each specific model in the family is defined by choosing suitable weights and kinematical decompositions in PP. In this way the exploration of the parameter space is extremely fast and complete. Summarising, each stellar density component in a multi-component model is characterised by different structural (density profile, flattening, total mass, scale-length), dynamical (rotational support, velocity dispersion anisotropy), and stellar population (age, metallicity, initial mass function, mass-to-light ratio) properties. The addition of a central $\mathrm{BH}$ and a DM halo is immediate.

The paper is organised as follows. Section 2 presents the analytical framework of the procedure, together with a new velocity decomposition for the azimuthal velocity field, to be used when the commonly adopted Satoh (1980) $k$-decomposition cannot be applied (a not uncommon case in multi-component systems). In Section 3 we detail how the scaling is carried out. In Section 4 some illustrative galaxy models are built, and a few tests are mentioned; we also present an application to the case of the exponential disc and its decomposition as sum of Miyamoto-Nagai discs. In Section 5 the main conclusions are summarised. Finally, Appendix A and B contain some relevant analytical details.

\section{MULTI-COMPONENT GALAXY MODELS}

In this section we introduce the general notation used, and we illustrate the main theoretical foundations on which our modeling procedure is based; in particular, we focus on the multi-component Jeans equations, on a generalisation of the Satoh $k$-decomposition for azimuthal motions, and on the projections on the plane of the sky. In the following Section 3, we will describe the scaling procedure, and in particular how the scaled solutions of the Jeans equations are obtained (with the Potential and Jeans Solver), and then combined by adopting suitable weights (in the PP phase).

\subsection{Structure of the galaxy models}

We adopt cylindrical coordinates $(R, \varphi, z)$, with the symmetry axis of the models aligned with the $z$-axis. In full generality, we consider models composed of $N$ different stellar density distributions $\rho_{* i}(R, z)$, of total mass $M_{* i}$, so that the total stellar density $\rho_{*}$ and the total stellar mass $M_{*}$ of the system are given respectively by

$\rho_{*}(R, z)=\sum_{i} \rho_{* i}, \quad M_{*}=\sum_{i} M_{* i}, \quad i=1, \ldots, N$.

From now on, sums over $i$ indicate sums over the $N$ stellar components. We assume that each $\rho_{* i}$ is made of a simple stellar population (see e.g. Renzini \& Buzzoni 1986; Maraston 2005), i.e. by stars of the same age, chemical composition, initial mass function, and in particular the same mass-to-light ratio $\Upsilon_{* i}$. Therefore, the total stellar distribution $\rho_{*}$ can be considered a composite stellar population; the luminosity density and the total luminosity of each stellar component can be written respectively as

$v_{* i}(R, z)=\frac{\rho_{* i}}{\Upsilon_{* i}}, \quad L_{i}=\frac{M_{* i}}{\Upsilon_{* i}}$,

so that

$v_{*}(R, z)=\sum_{i} v_{* i}, \quad L=\sum_{i} L_{i}$.

The local and average stellar mass-to-light ratios of the galaxy are given by

$\Upsilon_{*}(R, z) \equiv \frac{\rho_{*}}{v_{*}}=\frac{\sum_{i} \rho_{* i}}{\sum_{i} \rho_{* i} / \Upsilon_{* i}}, \quad<\Upsilon_{*}>\equiv \frac{M_{*}}{L}=\frac{\sum_{i} M_{* i}}{\sum_{i} M_{* i} / \Upsilon_{* i}}$,

where it is apparent how in general the local stellar mass-to-light ratio in a multi-component model depends on position.

From equation (1) the gravitational potential associated with the total stellar density is

$\phi_{*}(R, z)=\sum_{i} \phi_{* i}$,

where $\phi_{* i}(R, z)$ is the potential originated by the density component $\rho_{* i}$. The presence of a central $\mathrm{BH}$, of mass $M_{\mathrm{BH}}$, produces the potential

$\phi_{\mathrm{BH}}(r)=-\frac{G M_{\mathrm{BH}}}{r}, \quad r=\sqrt{R^{2}+z^{2}}$,

and an axisymmetric DM halo, of density $\rho_{\mathrm{h}}(R, z)$ and total mass $M_{\mathrm{h}}$ (when finite), produces the potential $\phi_{\mathrm{h}}(R, z)$. Therefore, in general, the total gravitational potential of the model is

$\Phi(R, z)=\phi_{*}+\phi_{\mathrm{h}}+\phi_{\mathrm{BH}}=\sum_{j} \phi_{j}, \quad j=1, \ldots, N+2$.

From now on, sums over $j$ indicate sums over all the $N+2$ galaxy components, i.e. the $N$ stellar components, the central $\mathrm{BH}$, and the DM halo. In principle, also the DM distribution can be made of different components, with a trivial generalisation of the current discussion, that is not necessary for the goal of this paper. Our scheme fully exploits the linearity of the Jeans equations with respect to the stellar density (Section 2.2) and to the gravitational potential (Section 3).

\subsection{The Jeans equations}

The procedure, in its basic version, assumes that each stellar component is implicitly described by a 2 -integral phase-space DF $f_{i}\left(E, J_{z}\right)$ (in general different for each component), where $E$ and $J_{z}$ are respectively the energy and the axial component of the angular momentum of each star (per unit mass) in the total potential $\Phi$. Therefore, the DF of the total stellar distribution is the 2-integral function

$f=\sum_{i} f_{i}$.

As usual, we indicate with $\left(\mathrm{v}_{R}, \mathrm{v}_{\varphi}, \mathrm{v}_{z}\right)$ the velocity components in the phase-space, and with a bar over a quantity the operation of average over the velocity-space. By construction, for each stellar component $\overline{\mathrm{v}}_{R}{ }_{i}=\overline{\mathrm{v}}_{z_{i}}=0$, the only non-zero ordered velocity can occur in the azimuthal direction $v_{\varphi i} \equiv \overline{\mathrm{v}}_{\varphi}$, and finally for the velocity dispersion tensor $\sigma_{R i}=\sigma_{z i} \equiv \sigma_{i}$. Of course, from equation (8) similar relations hold for the kinematical fields of the total $\rho_{*}$.

The Jeans equations for each stellar component are obtained as 
velocity averages of the Collisionless Boltzmann Equation over the corresponding $f_{i}$ (e.g. BT08), so that

$$
\left\{\begin{array}{l}
\frac{\partial \rho_{* i} \sigma_{i}^{2}}{\partial z}=-\rho_{* i} \frac{\partial \Phi}{\partial z}, \\
\frac{\partial \rho_{* i} \sigma_{i}^{2}}{\partial R}=\rho_{* i} \frac{\Delta_{i}}{R}-\rho_{* i} \frac{\partial \Phi}{\partial R},
\end{array}\right.
$$

where $\Phi$ is the total potential in equation (7), and

$\Delta_{i} \equiv \overline{\mathrm{v}}_{\varphi_{i}}^{2}-\sigma_{i}^{2}, \quad \sigma_{\varphi i}^{2}=\overline{\mathrm{v}}_{\varphi_{i}}^{2}-v_{\varphi i}^{2}=\Delta_{i}+\sigma_{i}^{2}-v_{\varphi i}^{2}$;

therefore, in the isotropic case, $\Delta_{i}=v_{\varphi i}^{2}$. Imposing the natural boundary condition $\rho_{* i} \sigma_{i}^{2} \rightarrow 0$ for $z \rightarrow \infty$, the solution of equations (9) is

$\rho_{* i} \sigma_{i}^{2}=\int_{z}^{\infty} \rho_{* i} \frac{\partial \Phi}{\partial z^{\prime}} d z^{\prime}, \quad \rho_{* i} \Delta_{i}=R\left(\frac{\partial \rho_{* i} \sigma_{i}^{2}}{\partial R}+\rho_{* i} \frac{\partial \Phi}{\partial R}\right)$.

We notice that $\Delta_{i}$ can be also recast as a commutator-like integral (e.g., see equation 35 in CMPZ21), with some advantage for analytical and numerical investigations; however we found by several numerical tests that $\Delta_{i}$ can also be accurately computed by (centred) numerical differentiation as in equation (11), and so in our code JASMINE2 we maintained this more direct way of evaluation.

A central point of the procedure is the sum rule in the phasespace imposed by the identity (8). In fact, with equation (8), we are assuming that the $N$ stellar components $\rho_{* i}$ are physically distinct, each of them described by its own $f_{i}$, and so necessarily the $N$ pairs of equations (9) are the moment equations of each $f_{i}$ in the total potential $\Phi$. As usual, if $F(\mathbf{x}, \mathbf{v})$ is a generic dynamical property defined over the phase-space, then

$\bar{F}_{i}=\frac{\int F f_{i} d^{3} \mathbf{v}}{\rho_{* i}}, \quad \bar{F}=\frac{\sum_{i} \rho_{* i} \bar{F}_{i}}{\rho_{*}}, \quad \bar{F} \mathscr{L}=\frac{\sum_{i} v_{* i} \bar{F}_{i}}{v_{*}}$,

where the properties $\bar{F}$ and $\bar{F} \mathscr{L}$ of the galaxy can be interpreted as the mass-weighted and the luminosity-weighted averages of the $\bar{F}_{i}$, respectively. Notice that we are not reconstructing here the phasespace DFs of the models; we just determine the general rules of combination of the velocity moments in multi-component systems. The previous considerations show how to combine the solution for the single $\rho_{* i}$ to obtain the dynamical fields associated with the total $\rho_{*}$. Clearly, the Jeans equations for $\rho_{*}$ in equation (1) are obtained as the sum of equations (9) over the $N$ components, and their solution can be written as

$\begin{cases}\sigma^{2}=\frac{\sum_{i} \rho_{* i} \sigma_{i}^{2}}{\rho_{*}}, & \Delta=\frac{\sum_{i} \rho_{* i} \Delta_{i}}{\rho_{*}}, \\ \overline{\mathrm{v}_{\varphi}^{2}}=\frac{\sum_{i} \rho_{* i} \mathrm{v}_{\varphi_{i}}^{2}}{\rho_{*}}, & v_{\varphi}=\frac{\sum_{i} \rho_{* i} v_{\varphi i}}{\rho_{*}},\end{cases}$

where the previous identities are of straightforward proof from equations (12) and (10). Note that $\sigma_{\varphi}^{2}$ is not given by the simple sum of the $\sigma_{\varphi i}^{2}$ of the single components, as $\sigma^{2}$ in equation (13) above, because from equations (13) and (10) one has:

$\sigma_{\varphi}^{2}=\overline{\mathrm{v}_{\varphi}^{2}}-v_{\varphi}^{2}=\Delta+\sigma^{2}-v_{\varphi}^{2}=\frac{\sum_{i} \rho_{* i}\left(\sigma_{\varphi i}^{2}+v_{\varphi i}^{2}\right)}{\rho_{*}}-v_{\varphi}^{2}$.

Similarly, from equation (12), we derive all the corresponding luminosity-weighted quantities; we do not give here their expressions, since they are just obtained by using as weights the luminosity densities $v_{* i}$ of the components instead of the mass densities $\rho_{* i}$, in equations (13) and (14).
Finally, the rotation curve in the equatorial plane is given in terms of the circular velocity $v_{\mathrm{c} j}$ of each mass component as

$v_{\mathrm{c}}^{2}=\sum_{j} v_{\mathrm{c} j}^{2}$

\subsection{Azimuthal velocity decomposition}

As well known, equations (9) are degenerate in the azimuthal direction, i.e. they only provide $\overline{\mathrm{v}}_{\varphi_{i}}^{2}=\sigma_{\varphi i}^{2}+v_{\varphi i}^{2}$. The most common phenomenological approach to break this degeneracy is the Satoh (1980) $k$-decomposition. If $\Delta_{i} \geq 0$ over the whole space, then one assumes

$v_{\varphi i}=k_{i} \sqrt{\Delta_{i}}, \quad \sigma_{\varphi i}^{2}=\sigma_{i}^{2}+\left(1-k_{i}^{2}\right) \Delta_{i}$,

with negative values of $k_{i}$ describing clockwise rotation. The special case $k_{i}^{2}=1$ corresponds to the isotropic rotator $\left(\sigma_{\varphi i}=\sigma_{i}\right)$, with flattening totally supported by rotation; while, if $k_{i}=0$, there is no net rotation $\left(v_{\varphi i}=0\right)$, and the flattening is totally supported by tangential velocity anisotropy. More general velocity decompositions can be obtained by assuming a position-dependent parameter $k_{i}(R, z)$, also allowing for values greater than unity, up to a positiondependent maximum determined by the request $\sigma_{\varphi i}=0$ (e.g. Satoh 1980; Ciotti \& Pellegrini 1996; Negri et al. 2014a). In principle each stellar component of a multi-component model is characterised by a different $k_{i}$, so that from equations (16) and (13) the total $\rho_{*}$ will have an effective Satoh parameter $k_{\mathrm{e}}$ given by

$k_{\mathrm{e}} \equiv \frac{v_{\varphi}}{\sqrt{\Delta}}=\frac{\sum_{i} k_{i} \rho_{* i} \sqrt{\Delta_{i}}}{\rho_{*} \sqrt{\Delta}}, \quad \sigma_{\varphi}^{2}=\sigma^{2}+\left(1-k_{\mathrm{e}}^{2}\right) \Delta$,

where $k_{\mathrm{e}}$ in general depends on position, even if the $k_{i}$ do not.

Clearly, in case of $\Delta_{i}<0$ for some $\rho_{* i}$, the Satoh decomposition in equation (16) cannot be applied. The case of a negative $\Delta_{i}$ over some regions of space (or everywhere) is not frequently encountered in applications, but it is not impossible; for example, it necessarily occurs for density components in multi-component systems with spherically symmetric total density, or in density distributions elongated along the symmetry axis (see e.g. Chapter 13 in Ciotti 2021). Indeed, $\Delta=0$ everywhere for a spherical system supported by a 2-integral DF, and thus, from equation (13), at least one $\Delta_{i}$ must be negative (excluding the trivial case of all the subcomponents spherically symmetric, so that $\left.\Delta_{i}=0\right)$. Notice that $\Delta_{i}<0$ is not necessarily a manifestation of an inconsistent $\mathrm{DF}\left(f_{i}<0\right)$, while if $\overline{\mathrm{v}_{\varphi_{i}}^{2}}=\Delta_{i}+\sigma_{i}^{2}<0$ certainly the whole model must be discarded as unphysical, even if the solution for the total stellar distribution are well-behaved. Therefore, if for some component $\Delta_{i}<0$, then in case of positivity of the sum $\Delta_{i}+\sigma_{i}^{2} \geq 0$, the Satoh decomposition is generalised to:

$v_{\varphi i}=k_{i} \sqrt{\Delta_{i}+\sigma_{i}^{2}}, \quad \sigma_{\varphi i}^{2}=\left(1-k_{i}^{2}\right)\left(\Delta_{i}+\sigma_{i}^{2}\right), \quad k_{i}^{2} \leq 1$,

where again $k_{i}$ can depend on position. We refer to this alternative decomposition as to the generalised $k$-decomposition. The case $k_{i}=0$ implies no net rotation $\left(v_{\varphi i}=0\right)$, while now $k_{i}^{2}=1$ corresponds to $\sigma_{\varphi i}=0$; notice that no isotropic rotators can be realised from equation (18) when $\Delta_{i}<0$, because isotropy $\left(\sigma_{\varphi i}=\sigma_{i}\right)$ would correspond to $k_{i}^{2}<0$. Moreover, while with the Satoh decomposition a spherical system cannot rotate and is isotropic independently of the value of $k_{i}$, with the generalised $k$-decomposition one can model rotating (and anisotropic) spherical systems. An application of this last case can be found, for example, in exploratory numerical simulations of rotating gas flows in galaxies of Yoon et al. (2019). Notice that the generalised decomposition applied to systems with 
$\Delta_{i} \gg \sigma_{i}^{2}$ (as for instance the case of highly flattened discs) reduces to the standard Satoh formula. A more interesting (and delicate) case, requiring particular care in the choice of the parameter $k_{i}$, is represented by systems with $\left|\Delta_{i}\right| \ll \sigma_{i}^{2}$, when we have $v_{\varphi i} \sim k_{i} \sigma_{i}$, and $\sigma_{\varphi i}^{2} \sim\left(1-k_{i}^{2}\right) \sigma_{i}^{2}$. This means that, in order to avoid substantial rotation, for example in almost spherical systems (oblate or prolate), $k_{i}$ must be kept small.

We finally remark that, for a given multi-component system, it is also possible to assume a Satoh decomposition for some components, and the generalised decomposition for the others; in analogy with equation (17) it is possible to define a total effective decomposition parameter $k_{\mathrm{e}}$ as

$k_{\mathrm{e}} \equiv \frac{v_{\varphi}}{\sqrt{\Delta+\sigma^{2}}}, \quad \sigma_{\varphi}^{2}=\left(1-k_{\mathrm{e}}^{2}\right)\left(\Delta+\sigma^{2}\right)$.

\subsection{Projections}

We recast here the projection formulae presented in Posacki et al. (2013) for the case of a multi-component system, focusing in particular on how the solutions for the components must be summed to obtain the projected fields of the total stellar distribution. We indicate with $<,>$ the scalar product, with $\mathbf{n}$ the line-of-sight direction (hereafter los) directed from the observer to the galaxy ${ }^{1}$, and with $l$ the integration path along the los. For the ease of notation in this Section we drop the subscript $i$, so that all the following formulae must be intended to hold separately for each stellar component $\rho_{* i}$ (and of course also for the total $\rho_{*}$ ). We will resume the use of the subscript $i$ at the end of the Section, when we give the expressions for the projected fields of $\rho_{*}$ as functions of the projected fields of the components. The projection of a stellar density, and of the ordered velocity $\boldsymbol{v}=v_{\varphi} \mathbf{e}_{\varphi}$, are

$\Sigma_{*}=\int_{-\infty}^{\infty} \rho_{*} \mathrm{~d} l, \quad \Sigma_{*} v_{\mathrm{los}}=\int_{-\infty}^{\infty} \rho_{*} v_{\varphi}<\mathbf{e}_{\varphi}, \mathbf{n}>\mathrm{d} l$,

where $\mathbf{e}_{\varphi}=(-\sin \varphi, \cos \varphi, 0)$ is the unitary vector in the tangential direction. From the adopted orientation of $\mathbf{n}$, a positive/negative $v_{\text {los }}$ indicates a motion receding from/approaching to the observer, respectively. The los velocity dispersion can be written as

$\sigma_{\text {los }}^{2}=\sigma_{\mathrm{P}}^{2}+V_{\mathrm{P}}^{2}-v_{\mathrm{los}}^{2}=V_{\mathrm{rms}}^{2}-v_{\mathrm{los}}^{2}$

(e.g. Ciotti \& Pellegrini 1996; Posacki et al. 2013, CMPZ21), where following Cappellari (2008) we also define $V_{\mathrm{rms}}^{2} \equiv \sigma_{\mathrm{P}}^{2}+V_{\mathrm{P}}^{2}$, and

$\Sigma_{*} \sigma_{\mathrm{P}}^{2}=\int_{-\infty}^{\infty} \rho_{*}<\sigma^{2} \mathbf{n}, \mathbf{n}>\mathrm{d} l$,

$\Sigma_{*} V_{\mathrm{P}}^{2}=\int_{-\infty}^{\infty} \rho_{*} v_{\varphi}^{2}<\mathbf{e}_{\varphi}, \mathbf{n}>^{2} \mathrm{~d} l$,

where in equation (22) $\sigma$ is the $3 \times 3$ velocity dispersion tensor. The fields $V_{\mathrm{rms}}$ and $v_{\mathrm{los}}$ in general depend on the specific direction $\mathbf{n}$, and $v_{\text {los }}, \sigma_{\mathrm{P}}$ and $V_{\mathrm{P}}$ on the specific velocity decomposition adopted, but $V_{\mathrm{rms}}$ is independent of the velocity decomposition. The previous identities are fully general and hold for a generic inclination of the los with respect to the galaxy. For our axisymmetric models, it is assumed without loss of generality that the los is parallel to the $x-z$ plane, and the projection plane rotates around the $y$ axis.

In particular, in the face-on projection (hereafter FO), the los is

1 At variance with the convention adopted in CMPZ21, where $\mathbf{n}$ points from the galaxy to the observer. parallel to the $z$ axis with $\mathbf{n}=-\mathbf{e}_{z}$, the projection plane is the $x-y$ plane, and

$\Sigma_{*}=2 \int_{0}^{\infty} \rho_{*} \mathrm{~d} z, \quad \Sigma_{*} \sigma_{\mathrm{los}}^{2}=2 \int_{0}^{\infty} \rho_{*} \sigma^{2} \mathrm{~d} z$

because $v_{\text {los }}=V_{\mathrm{P}}=0$, and so $\sigma_{\text {los }}=\sigma_{\mathrm{P}}$. In the edge-on projection (hereafter EO), the los is aligned with the $x$ axis with $\mathbf{n}=-\mathbf{e}_{x}$, the projection plane coincides with the $y-z$ plane, and $(\cos \varphi, \sin \varphi)=$ $(x / R, y / R)$ where $R=\sqrt{x^{2}+y^{2}}$. Then, from equation (20),

$\Sigma_{*}=2 \int_{y}^{\infty} \frac{\rho_{*} R}{\sqrt{R^{2}-y^{2}}} \mathrm{~d} R, \quad \Sigma_{*} v_{\text {los }}=2 y \int_{y}^{\infty} \frac{\rho_{*} v_{\varphi}}{\sqrt{R^{2}-y^{2}}} \mathrm{~d} R$.

Moreover, with some algebra, from equations (22) and (23) we have

$\Sigma_{*} \sigma_{\mathrm{P}}^{2}=2 \int_{y}^{\infty} \frac{\left(R^{2}-y^{2}\right) \sigma^{2}+y^{2} \sigma_{\varphi}^{2}}{R \sqrt{R^{2}-y^{2}}} \rho_{*} \mathrm{~d} R$,

$\Sigma_{*} V_{\mathrm{P}}^{2}=2 y^{2} \int_{y}^{\infty} \frac{\rho_{*} v_{\varphi}^{2}}{R \sqrt{R^{2}-y^{2}}} \mathrm{~d} R$,

so that, from equation (10), equation (21) can be recast in compact form as

$\Sigma_{*} \sigma_{\mathrm{los}}^{2}=2 \int_{y}^{\infty} \frac{R^{2} \sigma^{2}+y^{2} \Delta}{R \sqrt{R^{2}-y^{2}}} \rho_{*} \mathrm{~d} R-\Sigma_{*} v_{\mathrm{los}}^{2}$,

where the independence of $V_{\text {rms }}$ from the specific azimuthal velocity decomposition is apparent.

The projection formulae for a multi-component stellar system can now be easily obtained, for a generic los, just by considering how the intrinsic quantities add. From equations (1) and (13), and from equations (20)-(23), it is immediate to see that

$\Sigma_{*}=\sum_{i} \Sigma_{* i}, \quad v_{\mathrm{los}}=\frac{\sum_{i} \Sigma_{* i} v_{\mathrm{los} i}}{\Sigma_{*}}, \quad V_{\mathrm{rms}}^{2}=\frac{\sum_{i} \Sigma_{* i} V_{\mathrm{rms} i}^{2}}{\Sigma_{*}}$,

and $\sigma_{\text {los }}^{2}$ is given again by equation (21).

No difficulty is encountered in the construction of the luminosityweighted fields analogous to equations (29), by using the surface brightness distributions $I_{* i}=\Sigma_{* i} / \Upsilon_{* i}$, and $I_{*}=\sum_{i} I_{* i}$, so the projected stellar mass-to-light ratio $\Upsilon_{* \operatorname{los}} \equiv \Sigma_{*} / I_{*}$, defined in analogy with the local $\Upsilon_{*}$ in equation (4).

Summarising, we now have the framework needed to determine the solution of the Jeans equations once the solutions for the single components in the total potential are known. In the following Section we detail how the solution of each stellar component is obtained, thanks to the adopted scaling procedure.

\section{SCALING OF MULTI-COMPONENT MODELS}

We show here how, thanks to the full use of the scalings allowed by the Poisson and the Jeans equations, once a set of solutions is obtained for them, one can build an arbitrarily large family of models, just by combining the scaled solutions in this set with different weights; the scheme thus provides several galaxy models with almost no effort. The basic idea is elementary. We recognise that equations (9), at fixed total potential $\Phi$, are invariant for a mass scaling of the density $\rho_{* i}$, i.e. at fixed $\Phi$ the derived velocity fields would be independent of the value of $M_{* i}$. However, as $\Phi$ contains also $\phi_{* i}$, equations (9) obviously are not invariant to such scaling; nonetheless, the $N+2$ 
equations for $\rho_{* i}$ in the potentials $\phi_{j}$

$$
\left\{\begin{array}{l}
\frac{\partial \rho_{* i} \sigma_{i j}^{2}}{\partial z}=-\rho_{* i} \frac{\partial \phi_{j}}{\partial z}, \\
\frac{\partial \rho_{* i} \sigma_{i j}^{2}}{\partial R}=\rho_{* i} \frac{\Delta_{i j}}{R}-\rho_{* i} \frac{\partial \phi_{j}}{\partial R},
\end{array}\right.
$$

and their solutions

$\rho_{* i} \sigma_{i j}^{2}=\int_{z}^{\infty} \rho_{* i} \frac{\partial \phi_{j}}{\partial z^{\prime}} \mathrm{d} z^{\prime}, \quad \rho_{* i} \Delta_{i j}=R\left(\frac{\partial \rho_{* i} \sigma_{i j}^{2}}{\partial R}+\rho_{* i} \frac{\partial \phi_{j}}{\partial R}\right)$,

with $\rho_{* i} \sigma_{i j}^{2} \rightarrow 0$ for $z \rightarrow \infty$, do have important scaling properties that will be exploited in Section 3.1. We note that here and in the following the double subscript in $\sigma_{i j}^{2}$ does not refer to the tensorial nature of the velocity dispersion, but just identifies the solution of the $i$-th stellar component in the $j$-th potential component.

Leaving aside for the moment the scaling properties of equations (30) and (31), it is obvious that the sums

$\sigma_{i}^{2}=\sum_{j} \sigma_{i j}^{2}, \quad \Delta_{i}=\sum_{j} \Delta_{i j}$

are the solution of equations (9), as can be demonstrated, first by summing over $j$ the $N+2$ equations (30) and their solutions (31), and comparing the resulting expressions with equations (9) and (11), and then by proving that the solution of equation (9) is unique from the imposed boundaries.

An important point is in order here. Despite the apparent similarity of the decomposition of $\sigma_{i}^{2}$ and $\Delta_{i}$ performed in equations (30) over the $N+2$ potential components $\phi_{j}$, with the decomposition of $\sigma^{2}$ and $\Delta$ performed in equations (9) over the $N$ stellar components $\rho_{* i}$, there is a fundamental conceptual difference between the two decompositions. In fact, equations (9) are true moments of the Collisionless Boltzmann Equation obeyed by the distribution functions $f_{i}$ in the total potential, and so they have a sort of autonomous physical meaning; equations (30), instead, are just a mathematical decomposition over the different $\phi_{j}$ of the Jeans equations for $\rho_{* i}$. As a consequence, phase-space consistency arguments apply to the solution of equations (9), but not to $\sigma_{i j}^{2}$ and $\Delta_{i j}$ separately: as far as the fields $\sigma_{i}^{2}$ and $\Delta_{i}$ are physically acceptable, the model is also acceptable, independently of the specific properties ${ }^{2}$ of its components $\sigma_{i j}^{2}$ and $\Delta_{i j}$.

Finally, we recall the decomposition rule for the Virial Theorem (in its scalar form; the formulae can be easily extended to its tensorial form) of each stellar component:

$2 K_{* i}=-W_{i}=-\sum_{j} W_{i j}$,

where $K_{* i}$ is the kinetic energy of the $i$-th stellar component, and

$W_{i j}=-4 \pi G \int_{0}^{\infty} \int_{0}^{\infty} \rho_{* i}\left(R \frac{\partial \phi_{j}}{\partial R}+z \frac{\partial \phi_{j}}{\partial z}\right) R \mathrm{~d} R \mathrm{~d} z$.

2 The situation is somewhat similar to that faced when decomposing a positive density distribution over some prescribed set of functions (e.g, spherical harmonics), when the basis functions can present regions of negative densities.

\subsection{The scaling scheme}

We describe below how the scaling scheme works in general, with particular reference to its numerical implementation in JASMINE2, and to its logically distinct parts of the Potential and Jeans Solver and of the PP. We distinguish three groups of model parameters for the construction of a multi-component model, summarised in Table 1. In the first group there are the physical scales $M_{*}$ and $r_{*}$, i.e. the total stellar mass and its scale-length. All the density and potential components are made dimensionless by scaling them to the quantities

$\rho_{\mathrm{n}} \equiv \frac{M_{*}}{4 \pi r_{*}^{3}}, \quad \phi_{\mathrm{n}} \equiv \frac{G M_{*}}{r_{*}}$.

We note that it is convenient to normalise the $2 \mathrm{D}$ numerical grid to $r_{*}$, with $\tilde{R} \equiv R / r_{*}$, and $\tilde{z} \equiv z / r_{*}$. A scaled grid guarantees the same resolution, independently of the actual physical size of the model, measured by $r_{*}$. Incidentally, JASMINE2 has a bilogarithmic grid, with a few hundreds of points in $\tilde{R}$ and $\tilde{z}$, ranging from $\approx 10^{-5}$ or less at the origin, up to $\approx 10^{2}$ or more at the outer edge. Even though the physical scales are logically introduced first, the values of $M_{*}$ and $r_{*}$ (and so of $\rho_{\mathrm{n}}$ and $\phi_{\mathrm{n}}$ ) are fixed in the last step of the model construction, at the end of the PP (see Table 1). In this way, different physical realisations (in size and total mass) can be obtained for the same multi-component galaxy model.

In the second group of parameters there are the relative mass weights $\mathcal{R}_{i} \equiv M_{* i} / M_{*}, \mathcal{R}_{\mathrm{h}} \equiv M_{\mathrm{h}} / M_{*}, \mathcal{R}_{\mathrm{BH}} \equiv M_{\mathrm{BH}} / M_{*}$ of the different components, the mass-to-light ratios $\Upsilon_{* i}$, and the parameters $k_{i}$ appearing in equations (16) and (18) for the kinematical decomposition of the azimuthal motions. By definition

$\sum_{i} \mathcal{R}_{i}=1$

and in full generality we write

$\rho_{* i}=\rho_{\mathrm{n}} \mathcal{R}_{i} \tilde{\rho}_{* i}, \quad \rho_{\mathrm{h}}=\rho_{\mathrm{n}} \mathcal{R}_{\mathrm{h}} \tilde{\rho}_{\mathrm{h}}, \quad \tilde{\rho}_{*}=\sum_{i} \mathcal{R}_{i} \tilde{\rho}_{* i}$.

where $\tilde{\rho}_{* i}$ and $\tilde{\rho}_{\mathrm{h}}$ are the scaled density distributions, and $\tilde{\rho}_{*}=\rho_{*} / \rho_{\mathrm{n}}$ is the dimensionless total stellar density. Notice that from equation (35) the volume integrals of $\tilde{\rho}_{* i}$ over the whole dimensionless numerical grid evaluate to $4 \pi$ by construction. Similarly,

$\phi_{* i}=\phi_{\mathrm{n}} \mathcal{R}_{i} \tilde{\phi}_{* i}, \phi_{\mathrm{h}}=\phi_{\mathrm{n}} \mathcal{R}_{\mathrm{h}} \tilde{\phi}_{\mathrm{h}}, \phi_{\mathrm{BH}}=\phi_{\mathrm{n}} \mathcal{R}_{\mathrm{BH}} \tilde{\phi}_{\mathrm{BH}}$,

and so

$\Phi=\phi_{\mathrm{n}} \sum_{j} \mathcal{R}_{j} \tilde{\phi}_{j}, \quad W_{i}=M_{*} \phi_{\mathrm{n}} \mathcal{R}_{i} \sum_{j} \mathcal{R}_{j} \tilde{W}_{i j}$,

and

$\sigma_{i}^{2}=\phi_{\mathrm{n}} \tilde{\sigma}_{i}^{2}=\phi_{\mathrm{n}} \sum_{j} \mathcal{R}_{j} \tilde{\sigma}_{i j}^{2}, \quad \Delta_{i}=\phi_{\mathrm{n}} \tilde{\Delta}_{i}=\phi_{\mathrm{n}} \sum_{j} \mathcal{R}_{j} \tilde{\Delta}_{i j}$.

Finally, from the assumption of a constant mass-to-light ratio $\Upsilon_{* i}$ for each stellar component,

$v_{* i}=\rho_{\mathrm{n}} \frac{\mathcal{R}_{i}}{\Upsilon_{* i}} \tilde{\rho}_{* i}, \quad L_{i}=\frac{\mathcal{R}_{i}}{\Upsilon_{* i}} M_{*}$.

The values of the weights are chosen in PP (see Table 1), because a change in their values, and in the kinematical decompositions, does not require to recompute the potentials and solve again the Jeans equations. This possibility allows for a fast construction of different models belonging to the same family.

One family indeed is characterised by the choice of the third group 
Parameters of the scaling scheme

Potential and Jeans Solver

Structural parameters

\begin{tabular}{ll}
\hline Scaled stellar and DM densities & $\tilde{\rho}_{* i}, \tilde{\rho_{\mathrm{h}}}$ \\
Scale-length ratios & $\xi_{i}=\frac{r_{* i}}{r_{*}}, \xi_{\mathrm{h}}=\frac{r_{\mathrm{h}}}{r_{*}}, \ldots$ \\
Shape parameters & $q_{i}, q_{\mathrm{h}}, \ldots$ \\
\hline \hline
\end{tabular}

\begin{tabular}{ll}
\hline \hline Weights & Post-Processing \\
\hline Mass ratios & $\mathcal{R}_{i}=\frac{M_{* i}}{M_{*}}, \mathcal{R}_{\mathrm{h}}=\frac{M_{\mathrm{h}}}{M_{*}}, \mathcal{R}_{\mathrm{BH}}=\frac{M_{\mathrm{BH}}}{M_{*}}$ \\
Mass-to-light ratios & $\Upsilon_{* i}=\frac{M_{* i}}{L_{i}}$ \\
Kinematical decompositions & $k_{i}, \lambda_{i}, \delta_{i}$ \\
\hline \hline
\end{tabular}

Post-Processing

Physical Scales

Total stellar mass $\quad M_{*}$

Total stellar density scale-length $\quad r_{*}$

Table 1. The three main steps involved in the construction of a multicomponent model, listed from top to bottom in the order in which they are considered in a numerical implementation of the scaling scheme (as described in Section 3.1).

of parameters, to be performed at the beginning of the model construction: the structural parameters of the scaled density components $\tilde{\rho}_{* i}$ and $\tilde{\rho}_{\mathrm{h}}$, that in full generality we indicate with the symbols $\xi_{i} \equiv r_{* i} / r_{*}$ and $\xi_{\mathrm{h}} \equiv r_{\mathrm{h}} / r_{*}$ for the different scale-lengths, and with $q_{i}$ and $q_{\mathrm{h}}$ for other parameters that determine the shape of the scaled densities (for example the flattenings in case of ellipsoidal density distributions). The values of the structural parameters must be assigned in order to run the Potential and Jeans Solver (see Table 1), and in general a change in some of their values requires a new computation of the potentials and of the Jeans solutions.

\subsubsection{The Potential and Jeans Solver}

For a chosen set of values for the structural parameters, the scaled Jeans equations are obtained from equations (30) and equations (37) and (38). In practice, for $N$ assigned scaled stellar components $\tilde{\rho}_{* i}$ and a scaled dark matter halo $\tilde{\rho}_{\mathrm{h}}$, the Potential \& Jeans Solver first computes the scaled potentials $\tilde{\phi}_{* i}$ and $\tilde{\phi}_{\mathrm{h}}$, and then solves the $N \times(N+2)$ pairs of scaled equations (30), one for each $\tilde{\rho}_{* i}$ in the potential $\tilde{\phi}_{j}$ (including $\tilde{\phi}_{\mathrm{BH}}$ ), over the dimensionless grid $(\tilde{R}, \tilde{z})$; thus the scaled fields $\tilde{\sigma}_{i j}^{2}$ and $\tilde{\Delta}_{i j}$ are obtained. The possibility to solve equations (30) without choosing $\mathcal{R}_{i}$ and $\mathcal{R}_{j}$ is due to the fact that, on one hand, the weights $\mathcal{R}_{i}$ appear linearly in both sides of equations (30); on the other hand, $\tilde{\sigma}_{i j}^{2}$ and $\tilde{\Delta}_{i j}$ scale linearly with $\mathcal{R}_{j}$, once the boundary condition is fixed to zero at infinity.

The details of the numerical implementation of the computation of the potentials and of the solution of the Jeans equations are described in Posacki et al. (2013). Here we recall that the standard choice for the numerical computation of the potential in JASMINE2 is the integral formula

$\phi=-4 G \int_{0}^{\infty} R^{\prime} \mathrm{d} R^{\prime} \int_{-\infty}^{\infty} \frac{\rho\left(R^{\prime}, z^{\prime}\right) \mathrm{d} z^{\prime}}{\sqrt{\left(R+R^{\prime}\right)^{2}+\Delta z^{2}}} \mathbf{K}\left[\sqrt{\frac{4 R R^{\prime}}{\left(R+R^{\prime}\right)^{2}+\Delta z^{2}}}\right]$

where $\Delta z=z-z^{\prime}$, and $\mathbf{K}$ is the complete elliptic integral of the first kind (see e.g. BT08; Ciotti 2021), evaluated as a 2-dimensional integration over a staggered grid. However, for genuinely ellipsoidal models, the code can the use the faster Chandrasekhar formula (e.g. equation 2.140 in BT08; equation 2.21 in Ciotti 2021), and, for disc distributions, the integral formula based on Bessel functions (see Section 4.3 and Appendix B). As already remarked, the numerical evaluation of the potential is the most time consuming part of the construction of a model. For this reason JASMINE2 also contains a continuously updated library of analytical density-potential pairs available in the literature (and in some cases also based on homoeoidal expansion, see e.g. Ciotti \& Bertin 2005), so that one can choose between the numerical computation of the potential and (when available) the use of the analytical potential.

\subsubsection{The Post-Processing}

As described in the previous Section, for a given multi-component model of assigned $\tilde{\rho}_{* i}, \tilde{\rho}_{\mathrm{h}}$, and with a central $\mathrm{BH}$, the Potential \& Jeans Solver gives the solution $\tilde{\sigma}_{i j}^{2}$ and $\tilde{\Delta}_{i j}$ of the scaled form of equations (30). These solutions are then combined in PP, with the assignment of the mass ratios $\mathcal{R}_{j}$, so that the solution $\sigma_{i}^{2}$ and $\Delta_{i}$ of equations (9) for $\rho_{* i}$ is obtained, according to equations (32) and (40). At this stage, as discussed in Section 2.3, the PP performs a positivity check of $\tilde{\Delta}_{i}$ and $\tilde{\Delta}_{i}+\tilde{\sigma}_{i}^{2}$ : in case of negativity of the last quantity, a new choice of the weights $\mathcal{R}_{j}$ is made, until positivity is reached. If positivity cannot be obtained for acceptable choices of $\mathcal{R}_{j}$, then the multi-component model is discarded as unphysical.

Once the mass weights are assigned and the positivity check is passed, the PP requires the parameter $k_{i}$ for the kinematical decomposition, that gives the scaled azimuthal velocity fields $v_{\varphi i}$ and $\sigma_{\varphi i}$. In full generality, we define each decomposition parameter as

$k_{i}(R, z)=\lambda_{i} \delta_{i}(R, z)$,

where $\lambda_{i}$ is a constant weight, and $\delta_{i}(R, z)$ is a position-dependent function; the standard Satoh parameter is obtained with $\delta_{i}=1$ and $\lambda_{i}=k_{i}$. The benefit of this factorisation is due to the fact that the projection formula of $v_{\varphi i}$ in equation (20) for a given $\delta_{i}(R, z)$ scales with $\lambda_{i}$, so that we can set the value of $\lambda_{i}$ after having computed the projection integral. As projections represent the second most time-consuming step, the possibility to choose (and change) $\lambda_{i}$ after projections is a significant advantage. Note that, at variance with what happens for the fields $\tilde{\sigma}_{i}^{2}, \tilde{\Delta}_{i}, \tilde{v}_{\varphi i}^{2}$ and $\tilde{\sigma}_{\varphi i}^{2}$, the mass weights $\mathcal{R}_{j}$ enter the expression of $\tilde{v}_{\varphi i}$ under a square root (see equations 16 and 18). This implies that the $\mathcal{R}_{j}$ must be chosen before calculating the projections that use $v_{\varphi i}{ }^{3}$. In other words, the possibility to modify the values of $\mathcal{R}_{j}$ in PP, allowed by the " $i j$-decomposition", ends with the computation of the scaled fields in equations (40).

Once we have obtained the intrinsic and projected fields of each $\rho_{* i}$, the last steps are to combine them to calculate the total (massand luminosity-weighted) intrinsic and projected fields of $\rho_{*}$ (respectively from equations 13,14 , and equations $20,21,29$ ), and finally to choose the physical scales $M_{*}$ and $r_{*}$.

\subsection{Summary}

Summarising, a family of multi-component galaxy models is defined by the choice of $N$ scaled stellar density components $\tilde{\rho}_{* i}$, a

\footnotetext{
3 The effective radius $R_{\mathrm{e}}$ of the total stellar distribution is obviously another important quantity that cannot be obtained as a linear combination of the effective radii of the stellar components, and it can only be computed after the choice of the weights $\mathcal{R}_{i}$ and $\Upsilon_{* i}$.
} 
scaled DM halo $\tilde{\rho}_{\mathrm{h}}$, and a central BH. The Potential and Jeans Solver computes the associated scaled potentials $\tilde{\phi}_{j}$, and then solves the $N \times(N+2)$ Jeans equations (30) in their scaled form. In the subsequent $\mathrm{PP}$, specific values of the mass ratios $\mathcal{R}_{i}, \mathcal{R}_{\mathrm{h}}, \mathcal{R}_{\mathrm{BH}}$, of the mass-to-light ratios $\Upsilon_{* i}$, and of the kinematical decompositions with the parameters $k_{i}$, are fixed, thus defining a specific model in the same family. The solution of the Jeans equations for the total density distribution is recovered as (mass- or luminosity-) weighted sums of the scaled solutions, and the projections along a given line-of-sight are performed. The values of the total stellar mass $M_{*}$, and of its scale-length $r_{*}$, complete the construction of the model.

There are at least two significant advantages in this procedure, when compared with a straightforward integration of the Jeans equations for a multi-component galaxy model. First, the gravitational potentials of each stellar component and of the DM halo need not to be recalculated every time the weights are changed in PP; thus the run of the most time expensive part of is required just once for all the models in the same family. Second, the possibility to choose the weight parameters in PP allows for a fast exploration of the parameter space (that, for multi-component models, can be very large). Qualitatively, the $N \times(N+2)$ set of the $i j$-th scaled solutions of the Jeans equations for each $i$-th density component in each $j$-th potential component, can be interpreted as basis vectors that are successively linearly combined with different weights, to obtain a specific solution belonging to a family of multi-component models.

As a final remark, note that the procedure described so far can be extended to more general velocity decompositions. For example, it is straightforward to insert in it the Cappellari (2008) orbital anisotropy, where $\sigma_{R i}=b_{i} \sigma_{z i}$, with $b_{i}$ a constant parameter that can be different for each stellar component, and the underlying $i$-th DF depends on 3 integrals of motion.

\section{FOUR ILLUSTRATIVE MULTI-COMPONENT MODELS}

In order to illustrate the new features and potentialities of our procedure, as implemented in JASMINE2, we firstly describe in some detail the building of three multi-component galaxy models. All three models are made of two stellar distributions, to which a DM halo with a spherical Navarro-Frenk-White profile (Navarro et al. 1996, hereafter NFW) and a central supermassive BH are added. In the first model (hereafter JJE) the total spherical stellar profile and an ellipsoidal stellar component, both with a Jaffe (1983) profile, are assigned; if the dark mass is set to zero, this model reduces to the JJe models of CMPZ21. The second model (hereafter JHD) consists of an ellipsoidal Jaffe stellar density distribution, that represents a light stellar halo, coupled with a heavy Miyamoto-Nagai stellar disc (hereafter MN, Miyamoto \& Nagai 1975). In the third model (hereafter JLD), the ellipsoidal Jaffe component dominates, while a small MN inner disc is counter-rotating. These three models, are intended to represent features observed in real galaxies, but they are not designed to reproduce specific objects. Finally, we illustrate the comparison between an exponential disc and its representation via the sum of three MN discs, as proposed by Smith et al. (2015).

\subsection{The JJE models}

JJE models are a natural generalisation of JJe models presented in CMPZ21: as these latter describe quite well real elliptical galaxies, and several of their dynamical properties can be expressed in analytical form, they also represent an obvious test for JASMINE2.

To better appreciate the properties of JJE models, we recall the main properties (and limitations) of JJe models. These are constructed by assigning a total density $\rho_{*}$ following the axisymmetric ellipsoidal generalisation of the Jaffe model, and another axisymmetric ellipsoidal Jaffe distribution $\rho_{* 1}$, with different flattening, scalelength and total mass; in CMPZ21 the density distribution $\rho_{*}-\rho_{* 1}$ ( $=\rho_{* 2}$ in the current notation), is interpreted as a DM halo; finally, a central $\mathrm{BH}$ is added to the system. The analytical conditions on $\rho_{* 1}$ to guarantee the positivity of $\rho_{* 2}$ are given, and then the Jeans equations for $\rho_{* 1}$ are solved in analytical closed form, by using homoeoidal expansion, truncated at the linear order in the flattenings of $\rho_{*}$ and $\rho_{* 1}$. Albeit several properties of JJe models can be expressed in analytical form (making these models quite useful in numerical simulations of gas flows in galaxies, see e.g. Gan et al. 2019a,b), a few important shortcomings still affect them: i) the Jeans equations for $\rho_{* 1}$ are integrated in the homoeoidal expansion limit, retaining only linear terms in the flattenings, and they have not been studied for the difference component $\rho_{* 2}$; ii) projected kinematical fields of $\rho_{* 1}$ can be obtained in analytical form only as asymptotic formulae at the center and at large radii. JASMINE2 is then the obvious tool to address the two points above.

Here we generalise the JJe models to JJE models, by considering for the total $\rho_{*}$ an ellipsoidal Jaffe profile, of total mass $M_{*}$, scalelength ${ }^{4} \xi$, and flattening $q$ :

$\rho_{*}(R, z)=\frac{\rho_{\mathrm{n}} \xi}{q m^{2}(\xi+m)^{2}}, \quad m^{2}=\tilde{R}^{2}+\frac{\tilde{z}^{2}}{q^{2}}$.

Therefore, at variance with JJe models, in JJE models the total Jaffe mass distribution is purely stellar. We then consider another ellipsoidal Jaffe density profile, of total mass $M_{* 1}=\mathcal{R}_{1} M_{*}$, scale-length $r_{* 1}=\xi_{1} r_{*}$, and flattening $q_{1}$ :

$\rho_{* 1}(R, z)=\frac{\rho_{\mathrm{n}} \mathcal{R}_{1} \xi_{1}}{q_{1} m_{1}^{2}\left(\xi_{1}+m_{1}\right)^{2}}, \quad m_{1}^{2}=\tilde{R}^{2}+\frac{\tilde{z}^{2}}{q_{1}^{2}}$.

The second stellar component is then defined as

$\rho_{* 2}(R, z)=\rho_{*}(R, z)-\rho_{* 1}(R, z)$,

with $M_{* 2}=M_{*}-M_{* 1}=\left(1-\mathcal{R}_{1}\right) M_{*}=\mathcal{R}_{2} M_{*}$, in agreement with equation (36). Notice that $\rho_{* 2}$ is not an ellipsoid, unless $q_{1}=q$, and even in this case $\rho_{* 2}$ is not a Jaffe ellipsoid, unless $\xi_{1}=\xi$. As extensively discussed in CMPZ21, $\rho_{* 2}$ could be negative somewhere (and so unphysical) for some choices of $\mathcal{R}_{1}, \xi_{1}$ and $q_{1}$. Remarkably, the conditions required to assure $\rho_{* 2} \geq 0$ can be expressed as analytical (and simple) inequalities, as shown in Appendix A.

The stellar distribution $\rho_{*}$ is embedded in a NFW DM halo (spherically symmetric for simplicity), of mass $M_{\mathrm{h}}\left(r_{\mathrm{t}}\right)=\mathcal{R}_{\mathrm{h}} M_{*}$ enclosed within a truncation radius $r_{\mathrm{t}}$, scale-length $r_{\mathrm{h}}=\xi_{\mathrm{h}} r_{*}$, and concentration $c \equiv r_{\mathrm{t}} / r_{\mathrm{h}}$ :

$\rho_{\mathrm{h}}(r)=\frac{\rho_{\mathrm{n}} \mathcal{R}_{\mathrm{h}}}{f(c) s\left(\xi_{\mathrm{h}}+s\right)^{2}}, \quad \phi_{\mathrm{h}}(r)=-\phi_{\mathrm{n}} \mathcal{R}_{\mathrm{h}} \frac{\ln \left(1+s / \xi_{\mathrm{h}}\right)}{f(c) s}$

where $s \equiv r / r_{*}$, and $f(c)=\ln (1+c)-c /(1+c)$. We complete the model with a central $\mathrm{BH}$ of mass $M_{\mathrm{BH}}=\mathcal{R}_{\mathrm{BH}} M_{*}$.

Summarising, JJE models are determined, besides the total stellar mass and scale-length, $M_{*}$ and $r_{*}$, by the two parameters $\xi$ and $q$ for $\rho_{*}$, the five parameters $q_{1}, \xi_{1}, \mathcal{R}_{1}, \Upsilon_{* 1}, k_{1}$ for $\rho_{* 1}$, the two parameters $\Upsilon_{* 2}, k_{2}$ for $\rho_{* 2}$, the three DM parameters $\xi_{\mathrm{h}}, c, \mathcal{R}_{\mathrm{h}}$, and the BH mass weight $\mathcal{R}_{\mathrm{BH}}$ (see Table 2 for a specific JJE model). JASMINE2 further generalises JJE models, with the addition of a

\footnotetext{
4 In the spherical limit, and in the assumption of constant mass-to-light ratio, the Jaffe scale radius $r_{*}$ is related to the effective radius by $R_{\mathrm{e}} \simeq 0.75 r_{*}$.
} 


\begin{tabular}{ccc}
\hline \hline Model & $\rho_{* 1}$ & $\rho_{* 2}$ \\
\hline \hline JJE & Jaffe & $\rho_{*}(\xi=1, q=1)-\rho_{* 1}$ \\
& $\xi_{1}=0.1$ & - \\
& $q_{1}=0.8$ & - \\
& $\mathcal{R}_{1}=0.04$ & $\mathcal{R}_{2}=0.96$ \\
& $\Upsilon_{* 1}=2$ & $\Upsilon_{* 2}=6$ \\
& $k_{1}=0.5$ & $k_{2}=0.2$ \\
\hline JHD & Jaffe & MN \\
& $\xi_{1}=1$ & $\tilde{b}=0.1$ \\
& $q_{1}=0.8$ & $q_{2}=10$ \\
& $\mathcal{R}_{1}=0.3$ & $\mathcal{R}_{2}=0.7$ \\
& $\Upsilon_{* 1}=6$ & $\Upsilon_{* 2}=2$ \\
& $k_{1}=0.5$ & $k_{2}=0.8$ \\
\hline JLD & Jaffe & MN \\
& $\xi_{1}=1$ & $\tilde{b}_{2}=0.01$ \\
& $q_{1}=0.8$ & $q_{2}=10$ \\
& $\mathcal{R}_{1}=0.96$ & $\mathcal{R}_{2}=0.04$ \\
& $\Upsilon_{* 1}=6$ & $\Upsilon_{* 2}=2$ \\
& $k_{1}=0.5$ & $k_{2}(R, z)$ \\
\hline \hline
\end{tabular}

Table 2. The parameters for the stellar components of the illustrative JJE, JHD and JLD models (Sections 4.1 and 4.2). In the JJE model, the component $\rho_{* 2}$ is obtained as difference between a total spherical $(q=1)$ Jaffe profile $\rho_{*}$, with scale-length $\xi=1$, and a small and light Jaffe ellipsoidal component $\rho_{* 1}$ (as done for JJe models in CMPZ21). The standard Satoh $k$-decomposition in equation (16) for $\rho_{* 1}$, and the generalised $k$-decomposition in equation (18) for $\rho_{* 2}$, are adopted. In the JHD model, an ellipsoidal Jaffe distribution is coupled to a massive and quite flat $\left(q_{2}=a / b=10\right) \mathrm{MN}$ disc; in both components a generalised $k$-decomposition is adopted. In the JLD model, the ellipsoidal Jaffe component has the same flattening and size as in the JHD model, but the disc is significantly smaller, and counter-rotates in the inner regions, with the position-dependent Satoh parameter in equation (50), while a constant Satoh parameter is applied to the Jaffe component. In all models, the DM halo has a spherical NFW profile with $\xi_{\mathrm{h}}=2.6, c=10, \mathcal{R}_{\mathrm{h}}=20$, and the $\mathrm{BH}$ is defined by $\mathcal{R}_{\mathrm{BH}}=0.002$.

second DM component given by a shallow and very extended quasiisothermal halo, as useful in simulations of gas flows in galaxies residing in groups or clusters (Ciotti et al. in preparation).

\subsubsection{Tests}

We can use JASMINE2 in two different tests: we can give in input the homoeoidal expansion of the density-potential pairs, truncated at the linear order, and compare the numerical solution with that of CMPZ21, to check the importance of quadratic flattening terms in the solution of the Jeans equations for JJe models; and we can give in input the true ellipsoidal model, to check how well the homoeoidal expansion reproduces its internal dynamics. At the same time, the previous tests allow for an accuracy check of JASMINE2.

In the first test, we feed JASMINE2 with the homoeoidal expansion for $\rho_{*}$ and $\rho_{* 1}$, and we compare the numerical results of integration of the Jeans equations with the analytical results: we obtain excellent agreement for all the kinematical fields, better than a fraction of percent over the whole numerical grid, ranging from $\approx 10^{-5}$ to $\approx 70$ (in units of $r_{*}$ ). The results tend to be slightly more discrepant at increasing flattenings, as expected, since the analytical results in CMPZ21 are limited to the linear order in the flattenings, while JASMINE2 takes automatically into account also the second order terms when integrating the Jeans equations (due to the product between the density and the gradient of the potential). By increasing the numerical resolution in the central regions, and moving to smaller and smaller distances from the center, we also verify that the asymptotic formulae for the projected kinematical fields are also perfectly recovered numerically. This first tests adds confidence that JASMINE2 is working properly and with high accuracy, but also provides a further support that the (quite cumbersome) analytical formulae in CMPZ21 are actually correct ${ }^{5}$.

In a second test, we compare the numerical results of JASMINE2 for the true ellipsoidal JJe models with the analytical results in CMPZ21 obtained from homoeoidal expansion, therefore moving beyond the effect of second order approximation in the flattenings explored in the first test. We find that for relatively small flattenings (corresponding to E2-E3 galaxies) the homoeoidal expansion truncated at the linear order provides quite good results, even when compared with true ellipsoidal models, with the most significant discrepancy in the intermediate regions.

\subsubsection{Results for a JJE model}

We move now to illustrate the main properties of a specific JJE model (see Table 2). The total stellar distribution $\rho_{*}$ has a spherical Jaffe profile obtained from equation (44) with $\xi=1$ and $q=1$; this quite artificial case allows us to discuss some subtleties that can occur to the kinematical decomposition in multi-component systems. The stellar component $\rho_{* 1}$ is obtained from equation (45) with $\xi_{1}=0.1$, $q_{1}=0.8, \mathcal{R}_{1}$ is 0.04 , and $\Upsilon_{* 1}=2$, i.e. it is a quite small ellipsoidal distribution at the center of the galaxy; note that, from equation (A15), the maximum possible value of $\mathcal{R}_{1}$ is 0.08 . The component $\rho_{* 2}$ accounts for the remaining $96 \%$ of the total stellar mass of the galaxy, and $\Upsilon_{* 2}=6$, so that $\rho_{*}$ could represent an elliptical galaxy with a central and younger stellar system. We add the spherical NFW DM halo, given by equation (47), with $\xi_{\mathrm{h}}=2.6, c=10$, and $\mathcal{R}_{\mathrm{h}}=20$, so that the DM mass inside $R_{\mathrm{e}}$ (see Footnote 3 ) is $\approx 0.45$ of the total mass. Finally, in agreement with BH-galaxy scaling relations (see e.g. Kormendy \& Ho 2013), the mass of the central BH is fixed to $\mathcal{R}_{\mathrm{BH}}=0.002$.

In the three top panels of Figure 1, we show the density distribution of the scaled components $\tilde{\rho}_{* 1}$ and $\tilde{\rho}_{* 2}$, and of the total stellar density $\tilde{\rho}_{*}=\rho_{*} / \rho_{\mathrm{n}}$. Being this last spherical, and $\tilde{\rho}_{* 1}$ oblate, $\tilde{\rho}_{* 2}$ in its central regions is slightly prolate, and this will affect its kinematical fields, as anticipated in Section 2.3. Additional information on the model structure is provided in the first column of Figure 2: the top panel shows the radial profiles in the equatorial plane of $\mathcal{R}_{1} \tilde{\rho}_{* 1}$, $\mathcal{R}_{2} \tilde{\rho}_{* 2}, \tilde{\rho}_{*}$, and $\mathcal{R}_{\mathrm{h}} \tilde{\rho}_{\mathrm{h}}$. The total $\rho_{*}$ is almost coincident with $\rho_{* 2}$, except for the central regions, where $\rho_{* 1}$ and $\rho_{* 2}$ are comparable. The DM density $\rho_{\mathrm{h}}$ overcomes $\rho_{*}$ outside $\approx 0.5 R_{\mathrm{e}}$. The bottom panel shows the radial profiles in the equatorial plane of the contributions to the circular velocity due to the various mass components: the $\mathrm{BH}$ contribution is dominant in the inner regions, the DM in the outer regions, while at intermediate distances from the centre the resulting circular velocity is quite flat.

Similar trends can be seen in the radial profiles of the velocity fields in the equatorial plane of Figure 3, where, in the first column from top to bottom, we show the rotational velocity, the vertical velocity dispersion, and the azimuthal velocity dispersion, of $\rho_{* 1}$ and $\rho_{* 2}$, and the total mass-weighted and luminosity-weighted fields. Note that in

5 These tests are similar in the approach to those already performed with JASMINE by using the analytical results of Smet et al. (2015): we recall that the numerical integration of the potential in JASMINE2 uses the same routines of JASMINE. 

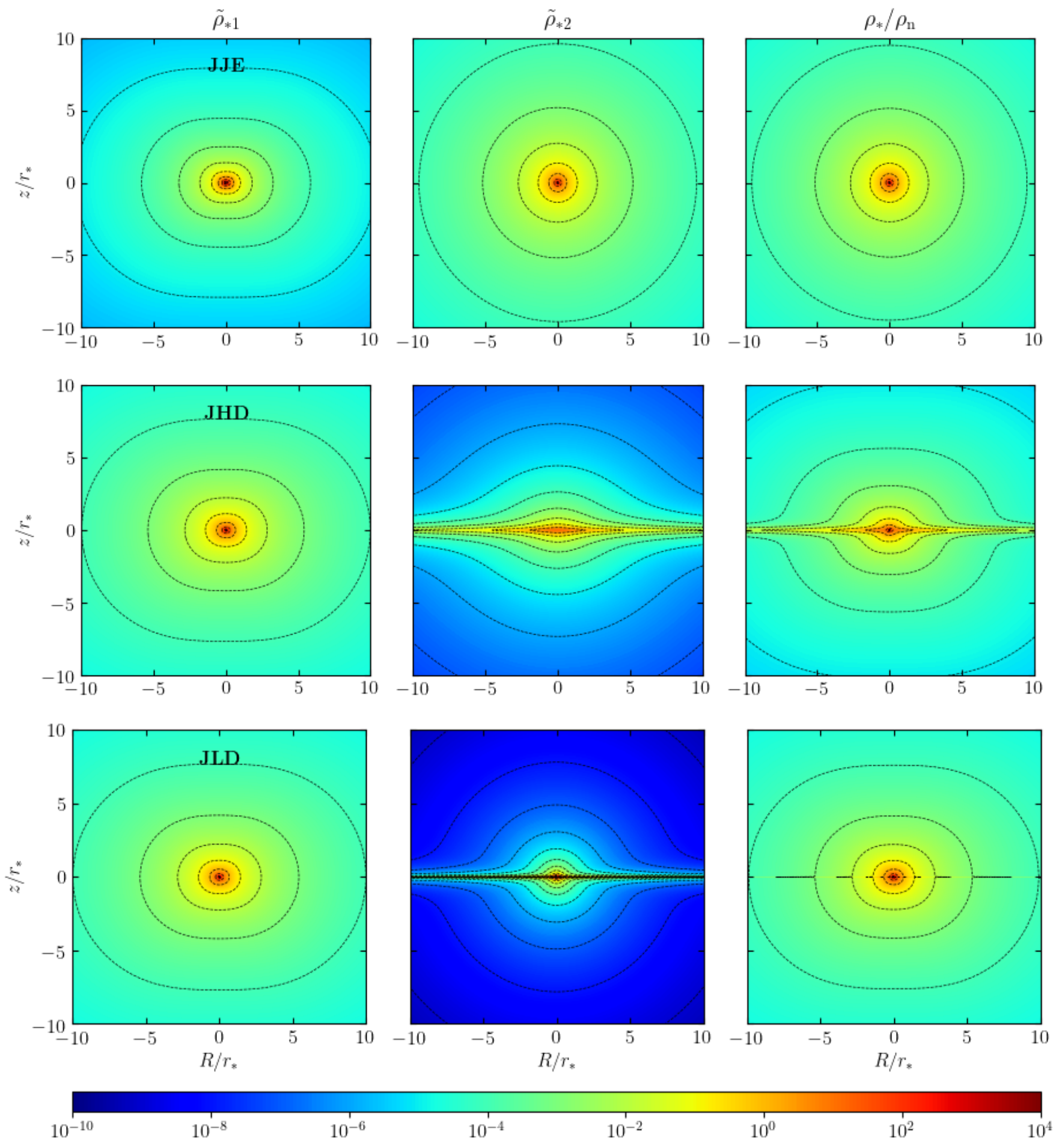

Figure 1. The scaled stellar distributions $\tilde{\rho}_{* 1}, \tilde{\rho}_{* 2}$, and the dimensionless total stellar distribution $\tilde{\rho}_{*}=\rho_{*} / \rho_{\mathrm{n}}$, of the three models of Table 2 . The dotted contours show the isodensities, with values spaced by 1 dex.

the three panels the vertical scale is the same, and the resulting system is clearly a slow rotator. This JJE model offers the opportunity to apply the generalised $k$-decomposition in equation (18); because the field $\Delta_{2}$, associated to the slightly prolate $\rho_{* 2}$, is negative in the central regions. As discussed in Section 2.3, we verified that $\overline{v_{\varphi_{2}}^{2}}$ is nowhere negative, and then we adopted the generalised decomposition, with a quite small $k_{2}=0.2$. The field $\Delta_{1}$ instead is everywhere positive, as expected, and so we adopted the standard Satoh formula with $k_{1}=0.5$. In the velocity profiles, the effect of the central $\mathrm{BH}$ is clearly visible; for example, the velocity dispersion profile of a Jaffe model with $\mathcal{R}_{\mathrm{BH}}=0$ would be constant in the central regions. Notice also how the velocity profiles, outside $\approx R_{\mathrm{e}}$, are almost coincident with the profiles of the more massive component $\rho_{* 2}$, in both the mass-weighted and luminosity-weighted cases; this is not surprising, 
JJE
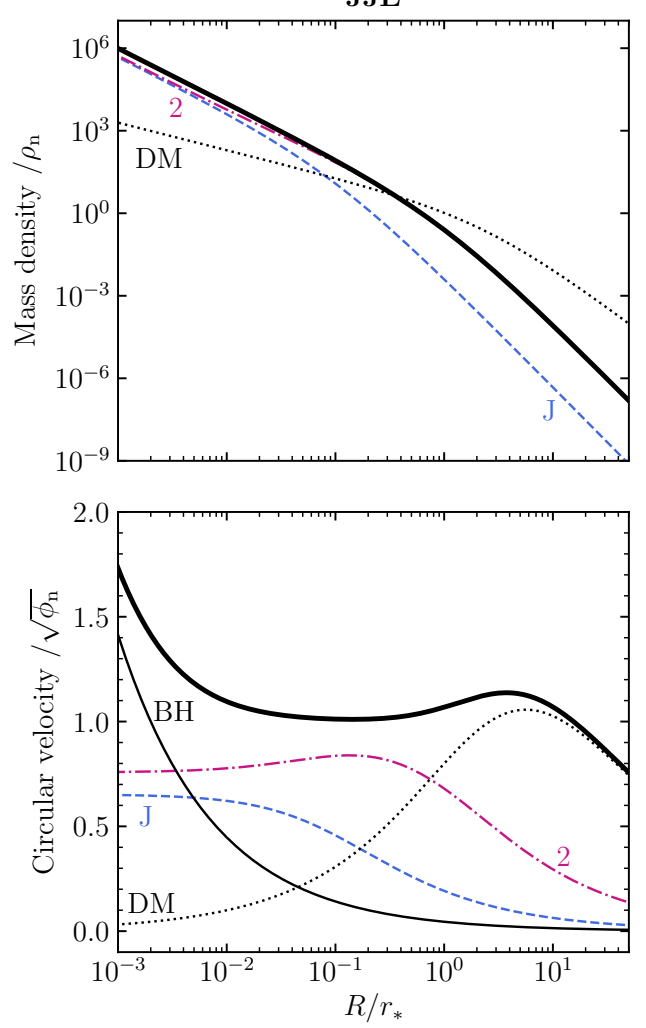

JHD
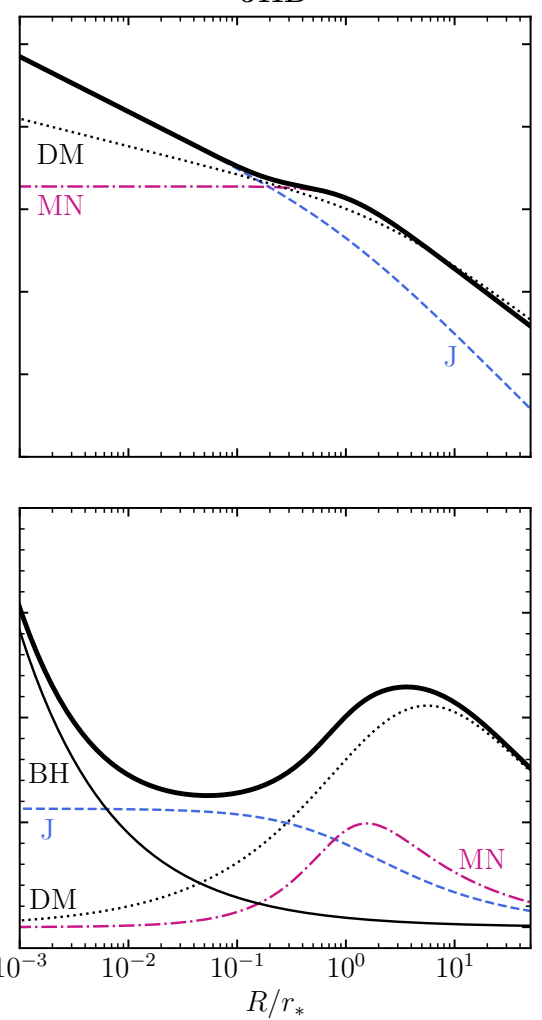

JLD
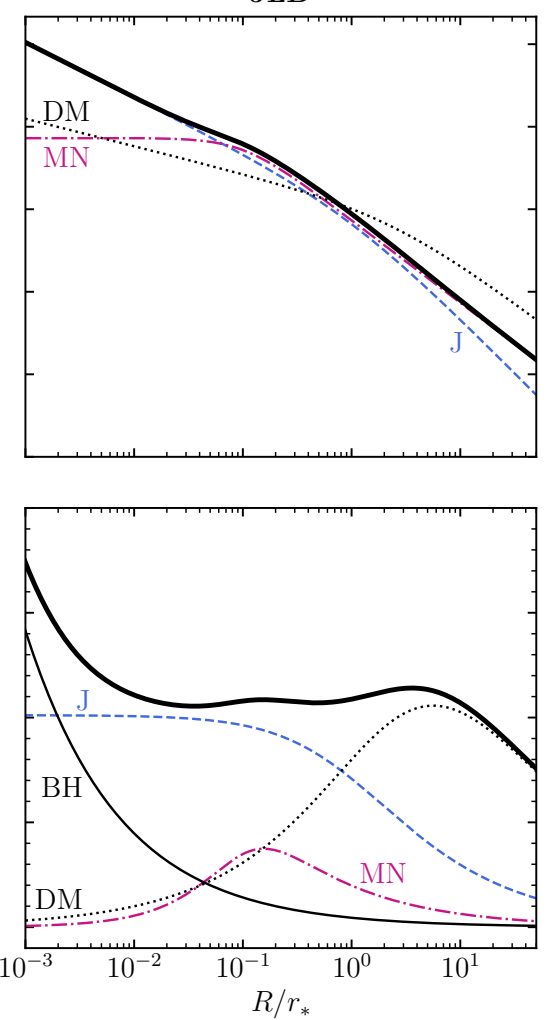

Figure 2. Radial profiles in the equatorial plane $(\tilde{z}=0)$ of the mass densities $\mathcal{R}_{1} \tilde{\rho}_{* 1}$ (dashed blue), $\mathcal{R}_{2} \tilde{\rho}_{* 2}$ (dotted-dashed magenta), $\tilde{\rho}_{*}$ (heavy solid), and $\mathcal{R}_{\mathrm{h}} \tilde{\rho}_{\mathrm{h}}$ (dotted), normalised to $\rho_{\mathrm{n}}$ (top row), for the three models of Table 2 . In the bottom row, we show the corresponding contributions to the total circular velocity (heavy solid) in the equatorial plane of the mass components, with the additional contribution of the central BH (solid), all normalised to $\sqrt{\phi_{\mathrm{n}}}$.

because in these regions $\rho_{*}$ coincides with $\rho_{* 2}$ (see Figures 1 and 2). The situation is different in the inner regions, where $\rho_{* 1}$ and $\rho_{* 2}$ are comparable: here the total velocities have intermediate values, with the luminosity-weighted profiles closer to the profiles of $\rho_{* 1}$ with the smaller mass-to-light ratio.

As an illustration of the projection procedure, in the first row of Figure 4, we show the EO projected luminosity-weighted fields $v_{\operatorname{los} \mathscr{L}}$ and $\sigma_{\operatorname{los} \mathscr{L}}$, with the superimposed dotted contours representing the galaxy isophotes of the surface brightness $I_{*}$. The slow rotation of the model is apparent from the colorbar values, indeed $v_{\operatorname{los}} \mathscr{L}$ is everywhere lower than $\sigma_{\operatorname{los} \mathscr{L}}$. A curious feature is the slightly vertically elongated shape of $\sigma_{\operatorname{los}} \mathscr{L}$ : this is not due to the prolate shape of $\rho_{* 2}$ in the central regions, but it is an effect of the generalised $k$-decomposition, coupled with the fact that $\Delta_{2}$ is almost null in the external regions, and so here $v_{\varphi 2} \sim k_{2} \sigma_{2}$, as introduced in Section 2.3. For example, an increase in $k_{2}$ would lead to an increase of the rotation in the external regions, with correspondent decrease of $\sigma_{\operatorname{los} \mathscr{L}}$, and with the net result of a more elongation of $\sigma_{\operatorname{los} \mathscr{L}}$ in the central regions.

\subsection{Ellipsoidal models with an embedded stellar disc}

JHD and JLD models consist of a stellar profile $\rho_{* 1}$ given again by the ellipsoidal Jaffe model in equation (45), coupled with a stellar MN disc $\rho_{* 2}$, of total mass $M_{* 2}=\mathcal{R}_{2} M_{*}$, and scale-lengths $a=\tilde{a} r_{*}$, $b=\tilde{b} r_{*}$ :

$\rho_{* 2}(R, z)=\rho_{\mathrm{n}} \mathcal{R}_{2} \tilde{b}^{2} \frac{\tilde{a} \tilde{R}^{2}+\left(\zeta+2 \sqrt{\tilde{z}^{2}+\tilde{b}^{2}}\right) \zeta^{2}}{\left(\tilde{R}^{2}+\zeta^{2}\right)^{5 / 2}\left(\tilde{z}^{2}+\tilde{b}^{2}\right)^{3 / 2}}$, $\phi_{* 2}(R, z)=-\frac{\phi_{\mathrm{n}} \mathcal{R}_{2}}{\sqrt{\tilde{R}^{2}+\zeta^{2}}}, \quad \zeta=\tilde{a}+\sqrt{\tilde{z}^{2}+\tilde{b}^{2}}$,

where $\mathcal{R}_{2}=1-\mathcal{R}_{1}$ from equation (36). For $a=0$ the $\mathrm{MN}$ disc reduces to the Plummer (1911) sphere, and for $b=0$ to the razorthin Kuzmin (1956) disc; in the following, we indicate with $q_{2}=a / b$ the disc flattening parameter. As in JJE models, we add the spherical NFW halo in equation (47), and a central $\mathrm{BH}$, so that the resulting multi-component models are completely determined once the values of $\xi_{1}, q_{1}, \mathcal{R}_{1}, \Upsilon_{* 1}, k_{1}$ for $\rho_{* 1}, \tilde{b}, q_{2}, \Upsilon_{* 2}, k_{2}$ for $\rho_{* 2}, \xi_{\mathrm{h}}, c, \mathcal{R}_{\mathrm{h}}$ for $\rho_{\mathrm{h}}$, and $\mathcal{R}_{\mathrm{BH}}$ for the $\mathrm{BH}$, are assigned, in addition to the total stellar mass $M_{*}$ and the scale length $r_{*}$ (see Table 2). In Section 4.2.1 we consider the case of a dominant MN disc, when the ellipsoidal Jaffe component can be interpreted as the stellar halo of a disc galaxy, and in Section 4.2.2 the case of a small and counter-rotating stellar disc at the center of a dominant stellar spheroid, as sometimes observed in real ETGs (e.g. Morelli et al. 2004; Krajnović et al. 2015; Mitzkus et al. 2017; see also Cappellari 2016). The parameters of the DM halo and of the central BH are the same as in the JJE model.

\subsubsection{Results for the JHD model}

In the "Jaffe - Heavy Disc" JHD model (see Table 2), the ellipsoidal Jaffe stellar halo $\rho_{* 1}$ is characterised by a scale-length $\xi_{1}=1$, a flattening $q_{1}=0.8$, a stellar mass fraction of $30 \%$ (i.e. $\mathcal{R}_{1}=0.3$ ), and a mass-to-light ratio $\Upsilon_{* 1}=6$. The dominant $\mathrm{MN}$ disc $\rho_{* 2}\left(\mathcal{R}_{2}=0.7\right)$ is quite flat $\left(q_{2}=10\right)$, with $\tilde{b}=0.1$, and a lower $\Upsilon_{* 2}=2$.

In the central row of Figure 1, the scaled density distributions 
JJE
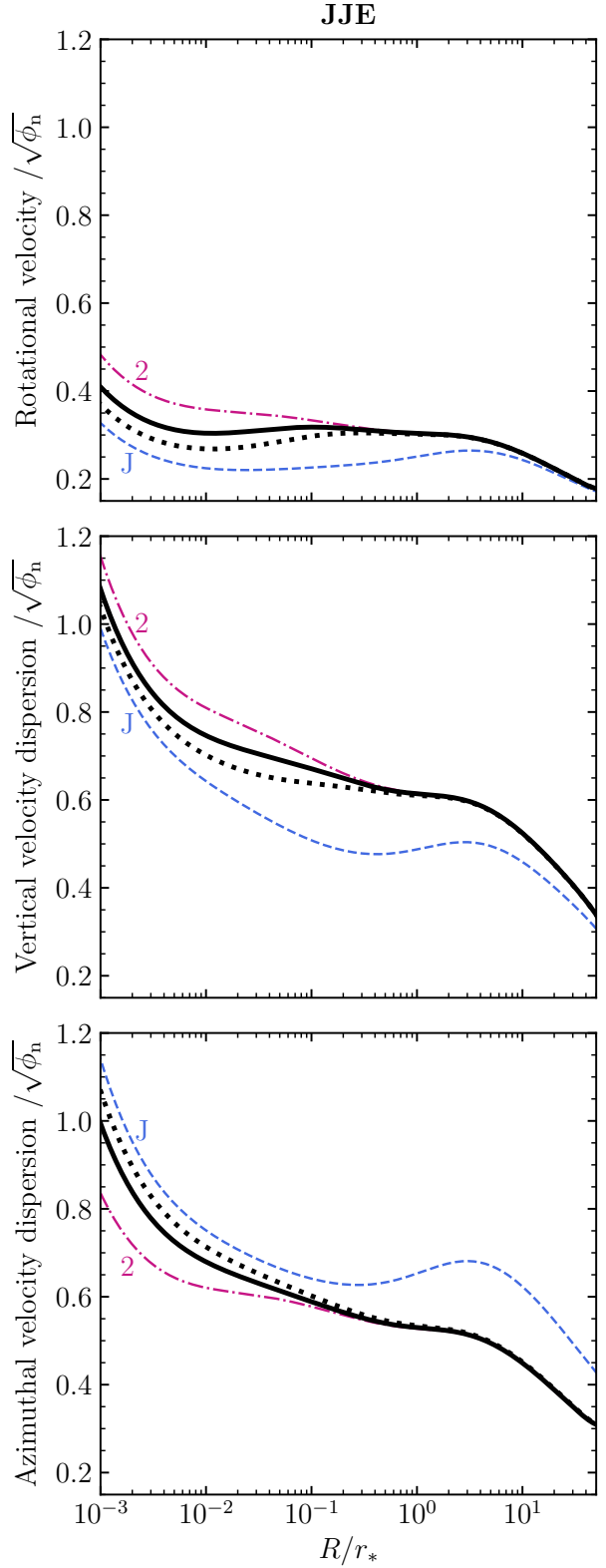

JHD
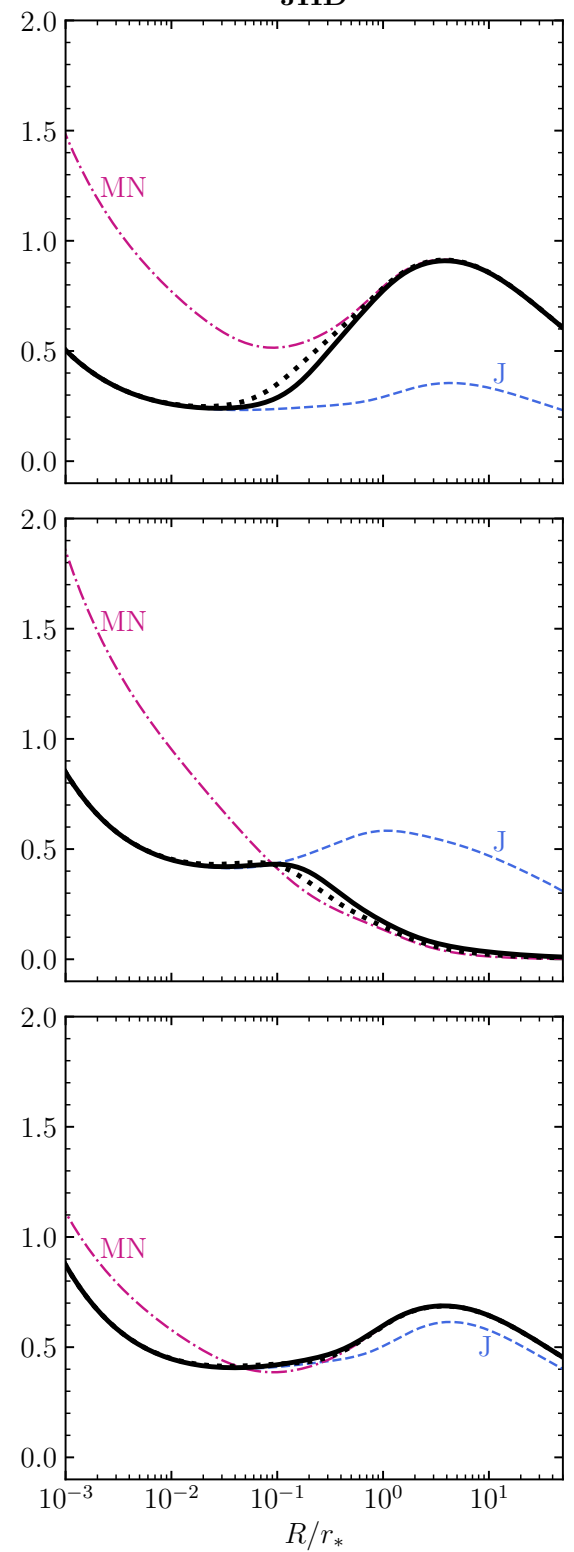

JLD
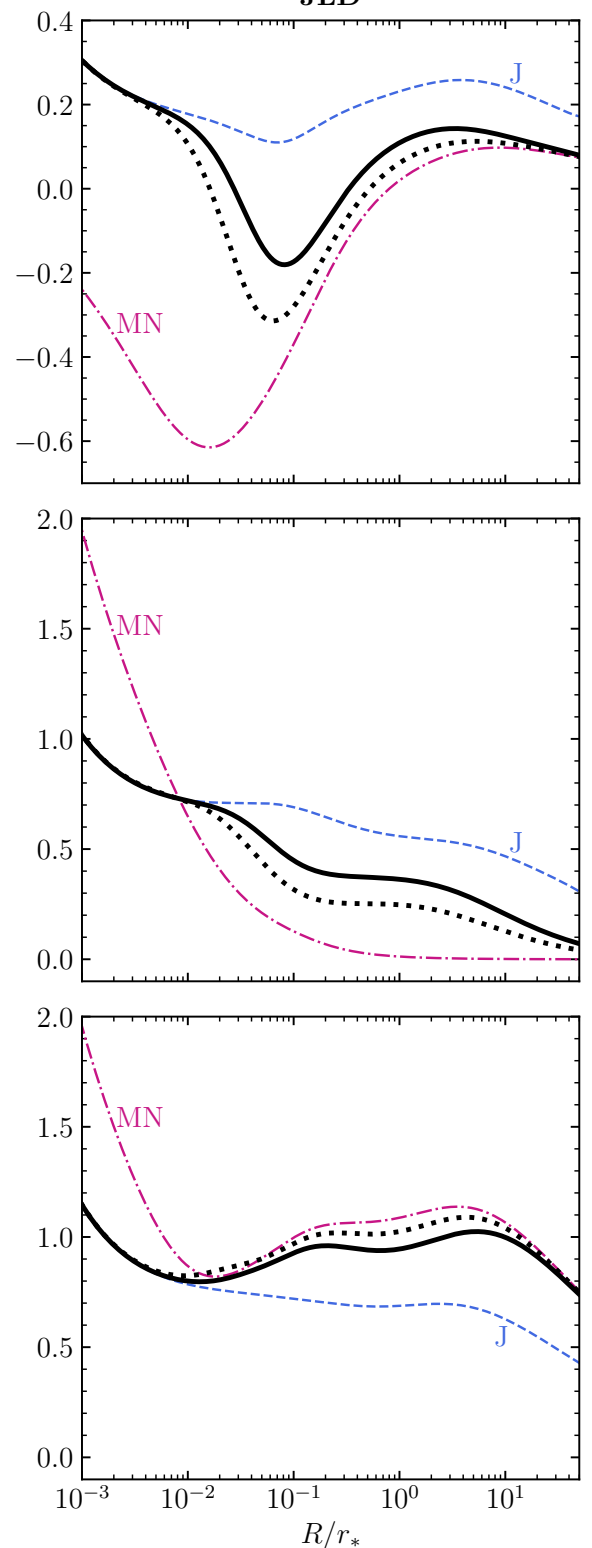

Figure 3. Radial profiles in the equatorial plane $(\tilde{z}=0)$ of the rotational velocities (top row), vertical velocity dispersions (middle row), and azimuthal velocity dispersions (bottom row), normalised to $\sqrt{\phi_{\mathrm{n}}}$, for the three models of Table 2. Each panel shows the total mass-weighted (heavy solid) and luminosity-weighted (heavy dotted) fields, together with the corresponding fields of $\rho_{* 1}$ (dashed blue) and $\rho_{* 2}$ (dotted-dashed magenta). Notice the different values on the vertical scales of the first column (JJE model) and of the top right panel showing the counter-rotation (JLD model).

$\tilde{\rho}_{* 1}, \tilde{\rho}_{* 2}$, and $\tilde{\rho}_{*}$, are shown. The resulting isodensity contours of $\rho_{*}$ would be classified as "discy" near the equatorial plane, and as "boxy" at large distance from the plane. The radial profiles of the density distributions (including the DM), in the equatorial plane, are shown in Figure 2. It is apparent how, inside $\approx 0.1 r_{*}$ the Jaffe halo dominates, around $r_{*}$ the MN disc dominates, and $\rho_{\mathrm{h}}$ overcomes the total $\rho_{*}$ outside $\approx 10 r_{*}$. Note that, even if $\mathcal{R}_{1}<\mathcal{R}_{2}, \rho_{* 1}$ dominates the total density in the central regions, due to the cuspy profile of the Jaffe density compared with the flat core of the MN density. The density decomposition reflects on the circular velocity profiles in the bottom panel of the same Figure: the total $v_{\mathrm{c}}$ at small radii is totally dominated by the $\mathrm{BH}$, and at large radii by the $\mathrm{DM}$ halo; while the "bump" around $3 r_{*}$ is due to the MN and the DM potentials.

The radial profiles in the equatorial plane of the velocity fields, obtained from the Jeans equations, are shown in the middle column of Figure 3, where from top to bottom the total mass- and luminosity-weighted rotational velocity, vertical velocity dispersion, and azimuthal velocity dispersion, are plotted together with the corresponding quantities for each stellar component separately. For the adopted values of the parameters in Table $2, \Delta_{1}$ turns out to be negative in a quite central region, while $\Delta_{1}+\sigma_{1}^{2}$ is everywhere positive; we decided to apply the generalised $k$-decomposition in equation (18) to both stellar components, with $k_{1}=0.5$ and $k_{2}=0.8$. The total velocity profiles, in the central regions, are completely determined by the Jaffe profile, because here $\rho_{* 1}>\rho_{* 2}$, compensating also for the higher $\Upsilon_{* 1}$; in the external regions, instead, the total profiles are dominated by the MN disc. Furthermore, $v_{\varphi}$ stays well below $v_{\mathrm{c}}$ both in the inner and outer regions (see $v_{\mathrm{c}}$ in Figure 2), as a clear 

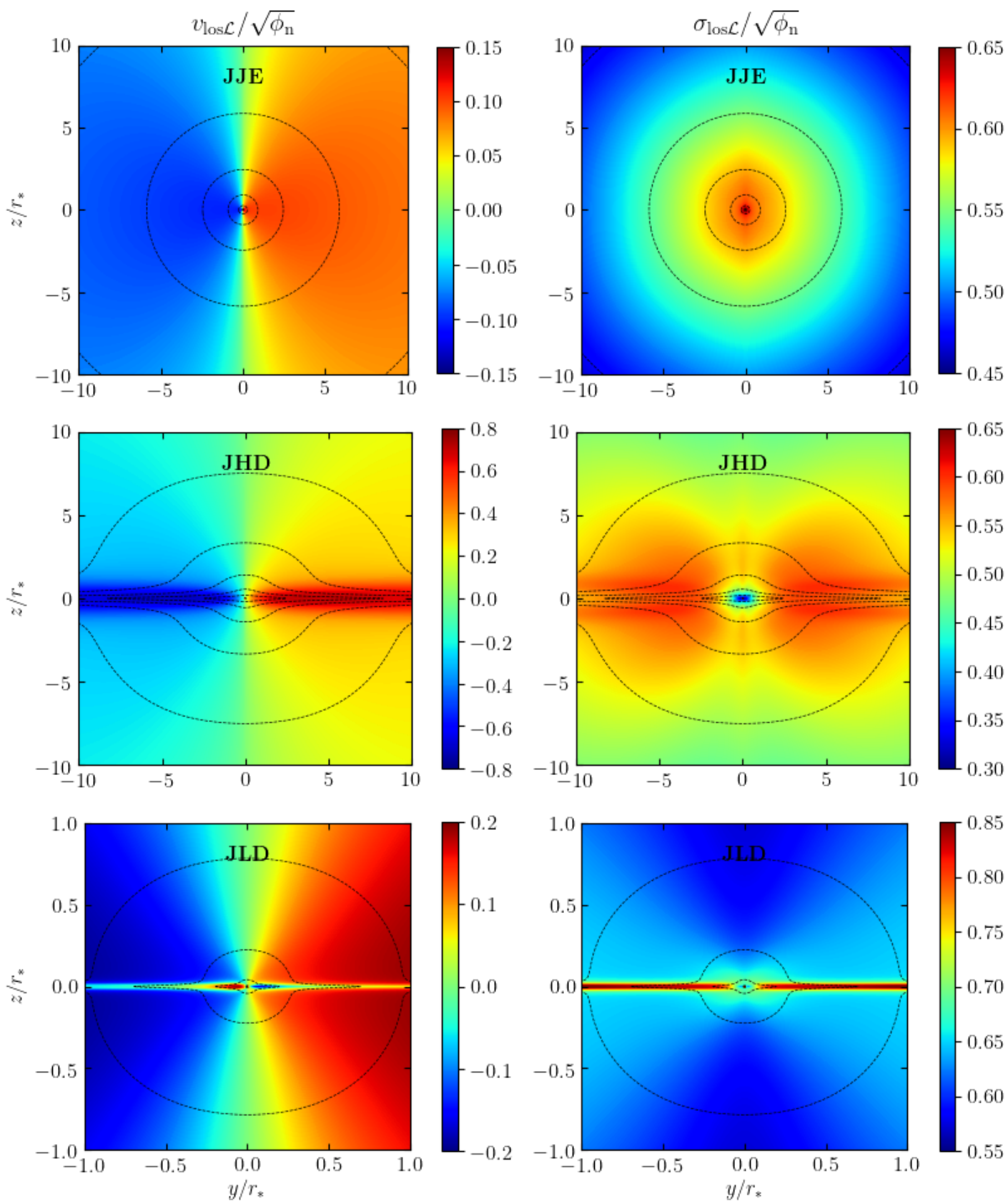

Figure 4. Edge-on projected luminosity-weighted rotational velocity $v_{\operatorname{los} \mathscr{L}}$ (left), and velocity dispersion $\sigma_{\operatorname{los} \mathscr{L}}$ (right), normalised to $\sqrt{\phi_{\mathrm{n}}}$, for the three models of Table 2. Notice the different ranges of values on the colorbars. For the JLD model, the region shown is limited to $r_{*}$ to appreciate the central features, in particular the inner counter-rotating disc. The dotted contours show the galaxy isophotes with values spaced by 1 dex.

manifestation of asymmetric drift in the equatorial plane (e.g. BT08). Note that $\sigma_{2}$, associated with a flat density profile at the centre, is much higher than $\sigma_{1}$, associated with $\rho_{* 1} \sim R^{-2}$ in the inner regions, as can be expected from the integration of the vertical Jeans equation for a power law density distribution in the gravitational field of a point-mass (i.e. the $\mathrm{BH}$ ). In addition, $\Delta_{2}$ of the $\mathrm{MN}$ model with the central BH vanishes at the centre (see e.g. Chapter 13 in Ciotti 2021), thus in the generalised $k$-decomposition, $v_{\varphi 2} \sim k_{2} \sigma_{2}$ (at variance with what would happen in the standard Satoh decomposition, i.e. $\left.v_{\varphi 2}=k_{2} \sqrt{\Delta_{2}}\right)$.

In the second row of Figure 4, the luminosity-weighted projected fields $v_{\operatorname{los} \mathscr{L}}$ and $\sigma_{\operatorname{los}} \mathscr{L}$ are shown, and the high rotation of the disc is clearly visible. The drop of $v_{\operatorname{los}} \mathscr{L}$ inside $r_{*}$ is due to a drop of the intrinsic rotational velocity (Figure 3 ). Also $\sigma_{\operatorname{los} \mathscr{L}}$ shows the highest values near the equatorial plane, with a toroidal distribution around the centre, and a drop inside $r_{*}$.

\subsubsection{Results for the JLD model}

At variance with the JHD model, in the "Jaffe - Light Disc" JLD model (see Table 2), the ellipsoidal Jaffe distribution $\rho_{* 1}$ accounts for almost the whole stellar mass of the galaxy $\left(\mathcal{R}_{1}=0.96\right)$, while its scale-length $\left(\xi_{1}=1\right)$, flattening $\left(q_{1}=0.8\right)$, and mass-to-light ratio $\left(\Upsilon_{* 1}=6\right)$ are unchanged. The component $\rho_{* 2}$ is a small MN disc, 
with $\tilde{b}=0.01$ and $\mathcal{R}_{2}=0.04$, while $q_{2}=10$ and $\Upsilon_{* 2}=2$ are the same of the JHD model.

The scaled density distributions, and the resulting total stellar density, are shown in the three bottom panels of Figure 1: $\tilde{\rho}_{* 1}$ is (structurally) identical to that of the JHD model, while $\tilde{\rho}_{* 2}$ is much more concentrated, so that the total stellar density is distributed in an extended halo with a very small disc. Indeed, the disc is almost invisible in the last panel, and it would be apparent only with a zoom in, as in Figure 4. The last column of Figure 2 shows the radial profiles in the equatorial plane of the density components, with their mass weights, and the resulting decomposition of the galaxy circular velocity profile. Notice that the central values of $\rho_{* 2}$ are higher than those in the JHD model, due to its smaller size, compensating for the reduced mass. In the circular velocity plot, this reflects into a larger contribution from the Jaffe component, and a smaller and inner "bump" of the $\mathrm{MN}$ component. As a result, $v_{\mathrm{c}}$ is almost flat between $10^{-2} r_{*}$ and $10 r_{*}$.

In the last column of Figure 3, the radial profiles of the velocity fields in the equatorial plane are shown. As in the previous models, of course, the total luminosity-weighted profiles, when distinguishable from the mass-weighted ones, are always closer to the profiles of the component with the smaller mass-to-light ratio. For the JLD model, both $\Delta_{1}$ and $\Delta_{2}$ are everywhere positive, so we apply the standard Satoh decomposition. The stellar halo is modeled as a slow rotator with $k_{1}=0.5$, while the circumnuclear stellar disc as a faster and counter-rotating light disc. In order to have counter-rotation limited to a central region, we adopt a position-dependent Satoh parameter, defined as follows:

$k_{2}(R, z)=k_{0}+\left(k_{\infty}-k_{0}\right) \frac{r}{r+0.1 r_{*}}, \quad r=\sqrt{R^{2}+z^{2}}$

(e.g. Ciotti et al. in preparation; see also Negri et al. 2014a for an alternative parametrisation), with $k_{0}=-0.8, k_{\infty}=0.1$, where the negative sign of $k_{0}$ assures the counter-rotation of the disc, as can be seen in the top right panel of Figure 3. At very small radii (inside $10^{-2} r_{*}$ ), the total rotational velocity is positive because the density is dominated by the Jaffe component. We stress that the module of $v_{\varphi 2}$ decreases towards the centre, at variance with the JHD model, because now $v_{\varphi 2}=k_{2} \Delta_{2}$, and $\Delta_{2} \rightarrow 0$, as explained in the previous Section. The central total vertical velocity dispersion is higher than that of the JHD model, even if the Jaffe component is structurally identical, because of the higher $\mathcal{R}_{1}$ and of the more concentrated MN disc.

In the last row of Figure 4, the los luminosity-weighted velocities show clearly the effect of the inner thin disc; the region shown is limited to $r_{*}$ to appreciate the central features. In particular, in the $v_{\operatorname{los} \mathscr{L}}$ distribution we have counter-rotation at small radii (but not in the very centre). The disc is also responsible for the highest values of the $\sigma_{\operatorname{los} \mathscr{L}}$ in the equatorial plane, and the extended surrounding toroidal distribution is also present, in analogy with the JHD model.

\subsection{Exponential discs and multi-MN decompositions}

Exponential discs are the common choice for modeling disc galaxies. Their gravitational potential can be constructed numerically by using the general formula based on complete elliptic integrals, or by using Bessel functions. The latter approach is particularly useful in case of factorised densities, such as

$\rho_{*}(R, z)=\rho_{0} \mathrm{e}^{-R / R_{\mathrm{d}}} V(|z| / h)$,

where the function $V$ describes the vertical structure of the disc, and $R_{\mathrm{d}}$ and $h$ are respectively its scale-length and scale-height; the razorthin exponential disc of central surface density $\Sigma_{0}$ is obtained for $V=$ $\delta(z / h)$ and $\rho_{0}=\Sigma_{0} / h$. Two natural generalisations of the infinitely thin exponential disc are obtained when $V$ is also an exponential function (double-exponential disc) or some negative power of the cosh function ("pseudo-isothermal" exponential disc).

Unfortunately, the gravitational potential of these discs cannot be obtained analytically; however, due to their relevance in the construction of galaxy models, alternative models with analytical potential have been proposed. In particular, the possibility to use multicomponent $\mathrm{MN}$ models to reproduce exponential discs, over some finite radial range, has been explored for example by Smith et al. (2015) and Rojas-Niño et al. (2016) (see also Flynn et al. 1996; Ciotti \& Pellegrini 1996). Such alternatives optimise the fit of the density profile, and produce a good agreement with the circular velocity profile of the exponential disc. Obviously, the superposition of $\mathrm{MN}$ discs with their power-law radial decay at large radii (equation (48)) cannot reproduce the exponential decay of equation (51). This forces to include at least one MN density component with negative mass (or negative scale-length), that can lead to a disc density distribution somewhere negative. The use of a multi-component MN representation of an exponential disc is motivated by the advantage of avoiding a time-consuming numerical computation of its gravitational potential. However, as we show in Appendix B, it is possible to obtain the potential of factorised exponential discs in equation (51) also with a very fast 1-dimensional integration in terms of Bessel functions (a method we implemented in JASMINE2).

As a last and natural application of our procedure, we extend the work carried out by Smith et al. (2015) by constructing the solutions of the Jeans equations for the double-exponential disc and for its everywhere positive density representation in terms of three $\mathrm{MN}$ discs (hereafter $3 \mathrm{MN}$ ). This $3 \mathrm{MN}$ decomposition is an ideal application of our modeling procedure, in particular because one MN component has negative mass, which gives the opportunity to illustrate how the scaling scheme in Section 3 works also with negative values of the mass ratios $\mathcal{R}_{i}$.

\subsubsection{Results for a double-exponential disc and its $3 M N$ fit}

We consider the single-component double-exponential model in equation (B3), with mass $\mathcal{R}_{\mathrm{d}}=M_{\mathrm{d}} / M_{*}=1$, scale-length $\alpha=$ $R_{\mathrm{d}} / r_{*}=1$, and scale-height $\beta=h / r_{*}=0.1$. For this density, we build the everywhere positive $3 \mathrm{MN}$ fit, following Section 2.2 in Smith et al. (2015). Accordingly, the three MN components (in our notation of equation 48 ) have the same scale-height $\tilde{b}$, but different $\mathcal{R}_{i}$ and scale-length $\tilde{a}_{i}(i=1,2,3)$; in particular, from their Figure 5, we obtain $\tilde{b}=0.12$, and from their equation (7) the values of $\mathcal{R}_{i}$ and $\tilde{a}_{i}$. The parameters for the double-exponential disc and for its $3 \mathrm{MN}$ fit are summarised in Table 3.

We compute the potential for the double-exponential disc both with the standard method in equation (42), and with the much faster integration of equation (B1), finding perfect agreement. As a safety check of the reconstructed $3 \mathrm{MN}$ model, we compare the circular velocity in the equatorial plane of the double-exponential disc and of its $3 \mathrm{MN}$ fit (Figure 5, top panel), that can be compared with Figure 3 of Smith et al. (2015), and the FO surface density profiles of the two models (Figure 5, bottom panel) that in turn can be compared with their Figure 7. The circular velocity of the exponential disc is almost perfectly reproduced over the explored radial range, while the reproduction of the FO surface density is less satisfactory, an unavoidable consequence of the everywhere positive decomposition adopted. For completeness, in Figure 6, we present the EO surface density distributions of the two models. As expected, the two distributions are quite different in the outer regions, especially for increasing distance 


\begin{tabular}{|c|c|c|c|}
\hline \multicolumn{4}{|l|}{ Model } \\
\hline Double-Exponential disc & & $\begin{array}{c}\alpha=R_{\mathrm{d}} / r_{*}=1 \\
\beta=h / r_{*}=0.1 \\
\mathcal{R}_{\mathrm{d}}=1 \\
k=1\end{array}$ & \\
\hline \multirow[t]{2}{*}{ 3MN fit (Smith et al. 2015) } & & $\tilde{b}=0.12$ & \\
\hline & $\begin{aligned} q_{1} & =4.64, \\
\mathcal{R}_{1} & =0.16,\end{aligned}$ & $\begin{array}{c}q_{2}=21.42 \\
\mathcal{R}_{2}=-5.77 \\
k=1\end{array}$ & $\begin{array}{c}q_{3}=18.67 \\
\mathcal{R}_{3}=6.72\end{array}$ \\
\hline
\end{tabular}

Table 3. The parameters of the double-exponential disc and its $3 \mathrm{MN}$ fit from Smith et al. (2015). For the meaning of the parameters of the doubleexponential disc, see Section 4.3.1 and Appendix B. For the 3MN model, we adopt the same notation of Section 4.2, with the same $\tilde{b}$ for all the three components, $q_{i}=\tilde{a}_{i} / \tilde{b}$, and $\mathcal{R}_{i}=M_{* i} / M_{*}$. The Jeans equations are solved in the isotropic case, with constant Satoh parameter $k=1$.

from the equatorial plane, where the $3 \mathrm{MN}$ model produces higher surface density values. Consequently, also the kinematical fields obtained from the solution of the Jeans equations are expected to show significant differences, especially at high $|z|$.

We use our procedure in JASMINE2 to evaluate these differences, a problem left open by the studies of Smith et al. (2015) and RojasNiño et al. (2016); we adopt for simplicity the case of the isotropic rotator, without a DM halo and a central BH. In Figure 7, the EO projected rotational velocity and velocity dispersion are shown. The fields $v_{\text {los }}$ of the two models look remarkably similar, also outside the equatorial plane. In particular, the percent error of the $3 \mathrm{MN}$ model with respect to the double-exponential model, in the equatorial plane, is $<9 \%$ out to $4 R_{\mathrm{d}}$, and $<14 \%$ out to $10 R_{\mathrm{d}}$. This quite satisfactory result is not obvious a priori, since $v_{\varphi}$, at variance with $v_{\mathrm{c}}$, is not a function of the potential only, but it also depends on the velocity dispersion via the asymmetric drift. Therefore, the excellent agreement of $v_{\mathrm{c}}$ in Figure 5 is not a guarantee that also $v_{\varphi}$, and its projection $v_{\text {los }}$, are well reproduced by the $3 \mathrm{MN}$ density fit. The reproduction of $v_{\text {los }}$ outside the equatorial plane is still quite good, with a slightly higher discrepancy at increasing $|z|$, as expected, but improving for larger galactocentric distances; for example, at $z=R_{\mathrm{d}}$, the percent error is $<23 \%$ out to $4 R_{\mathrm{d}}$, reducing to $<18 \%$ out to $10 R_{\mathrm{d}}$. The situation is different for $\sigma_{\text {los }}$ : the two fields are significantly different, even in the equatorial plane, with the $3 \mathrm{MN}$ model showing values up to a factor of 2 larger than those of the double-exponential model. Moreover, the velocity dispersion of the $3 \mathrm{MN}$ model near the rotation axis presents a characteristic hourglass-shaped distribution (see also Negri et al. 2014a), only barely detectable at the very centre for the double-exponential model. Notice that this feature is not observed in the maps of Figure 4 for the JHD and JLD models, even if they also contain a MN component, due to the addition of a stellar halo and a DM halo, and to the different kinematical decompositions adopted (see also the discussion in Smet et al. 2015). These experiments suggest caution when adopting the $3 \mathrm{MN}$ representation to interpret the observed velocity dispersion of disc galaxies.

\section{CONCLUSIONS}

We presented the theoretical framework for an efficient Jeans modeling of multi-component axisymmetric galaxies, and its numerical implementation in the code JASMINE2, significantly upgraded from its original version JASMINE (Posacki et al. 2013). In this framework the models can include an arbitrary number of stellar
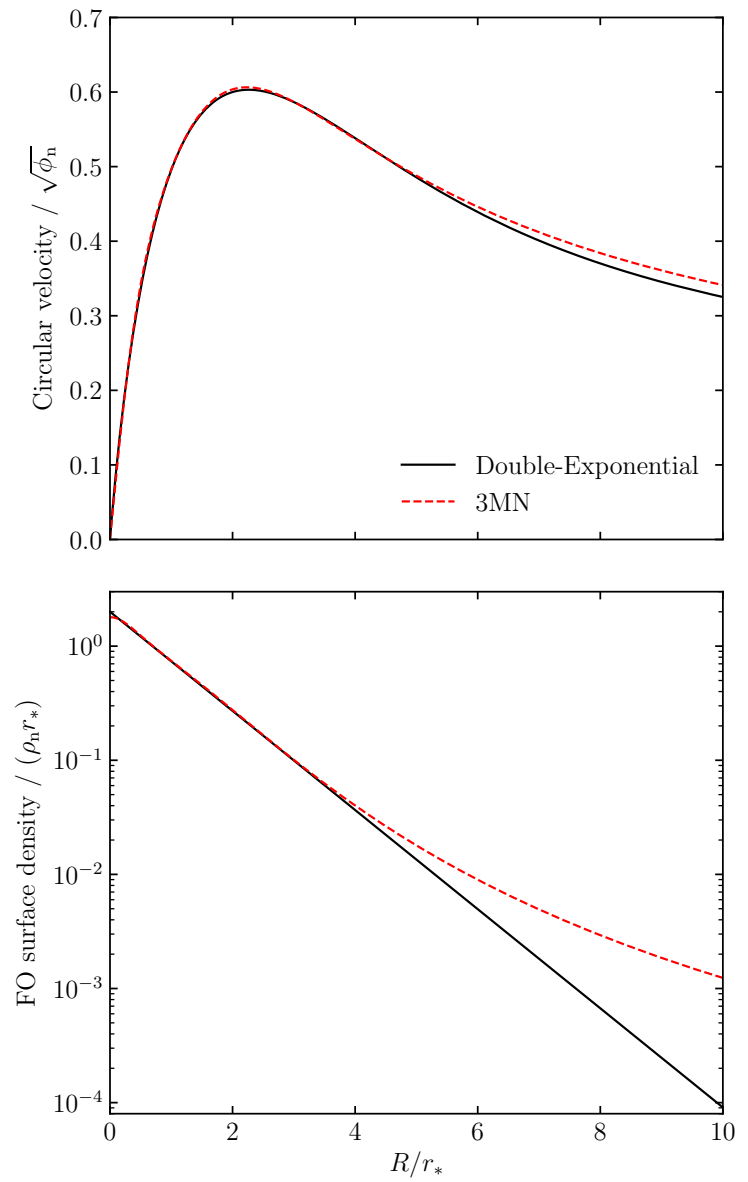

Figure 5. Circular velocity (top) and face-on surface density (bottom) profiles of the two models of Table 3; these plots can be compared with Figures 3 and 7 in Smith et al. (2015).

components, with different structural, dynamical and stellar population properties, a DM halo, and a central BH. The structural and dynamical properties of each stellar component can be mass- or luminosity-weighted, and projected on the plane of the sky. The internal dynamics of each stellar component is implicitly described by a 2-integral DF (in general different for each component), so that a phenomenological decomposition of the azimuthal velocity field must be chosen. For each component, we can adopt the Satoh (1980) $k$-decomposition, where $v_{\varphi i}=k_{i} \sqrt{\Delta_{i}}$, or a generalised $k$ decomposition, where $v_{\varphi i}=k_{i} \sqrt{\Delta_{i}+\sigma_{i}^{2}}$; furthermore, the parameter $k_{i}$ can be constant or position-dependent. The generalised decomposition allows for the modeling of systems with $\Delta_{i}<0$ (as may happen for density distributions elongated along the symmetry axis). The presented scheme can be easily extended to more general velocity decompositions, such as that introduced by Cappellari (2008), where the underlying DF depends on 3 integrals of motion.

In the numerical implementation, the gravitational potential of the density components is computed by default in terms of complete elliptic integrals (equation 42), a very accurate but quite time-expensive approach, that can become impractical especially when dealing with the exploration of the parameter space of multi-component models. To reduce the computational time, we fully exploited the scalings allowed by the Poisson and the Jeans equations, and by the projection formulae. The resulting scheme led to an organisation of JASMINE2 in two logically distinct parts: the Potential and Jeans Solver and 


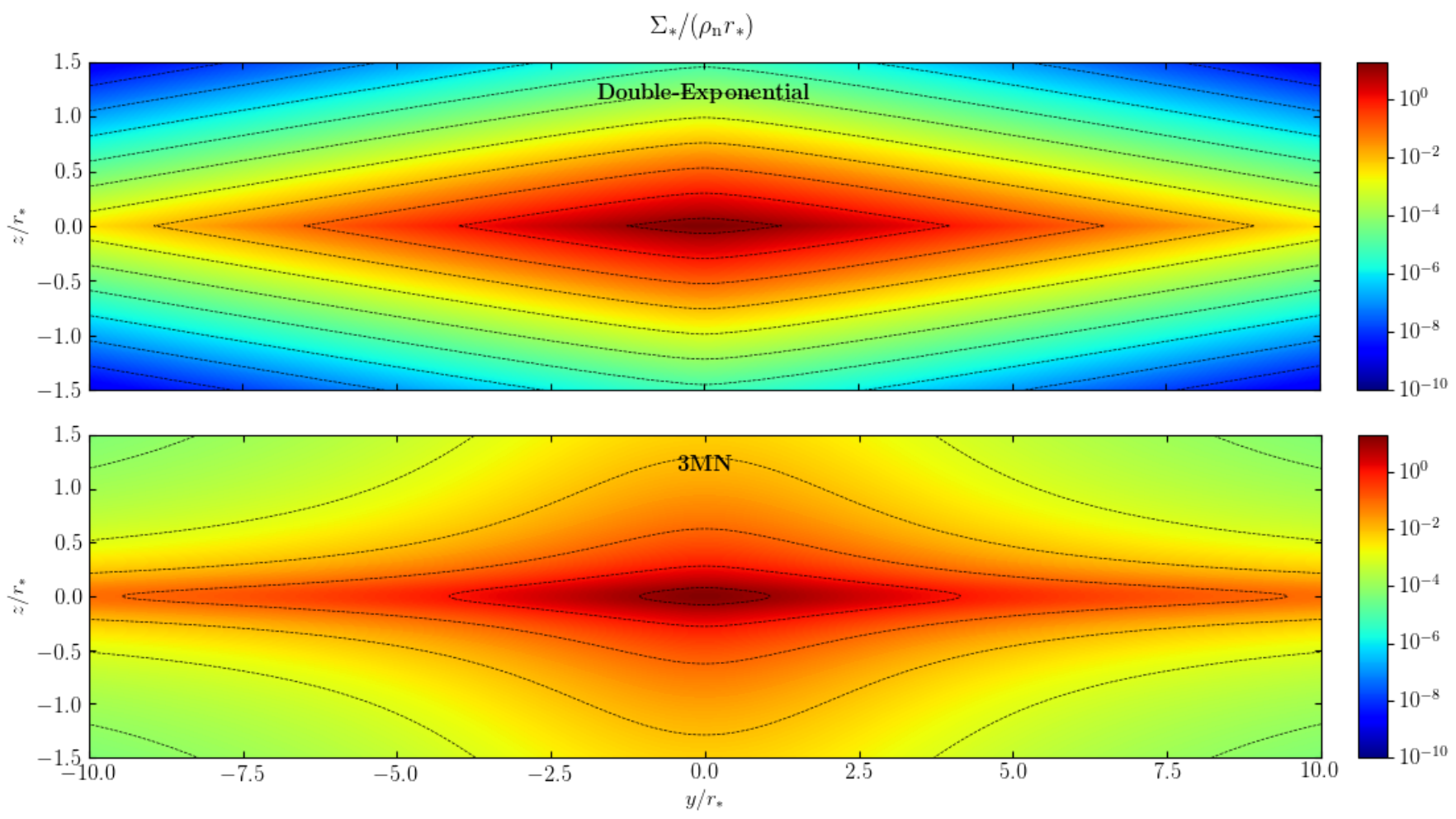

Figure 6. Edge-on surface density distributions $\Sigma_{*}$ of the two models of Table 3. The dotted contours are spaced by 1 dex.

the Post-Processing (PP). In practice, once the structural properties of the scaled stellar and DM distributions are assigned, the code computes, with a single run of the Potential and Jeans Solver, the scaled solutions of the Jeans equations, defining a family of models. The scaled Jeans solutions are then combined in PP, with the desired mass and luminosity weights, and the choice of appropriate kinematical decompositions, and then projected. The PP procedure can be performed several times, obtaining different specific models in the same family. Finally, for each model, the two physical scales $M_{*}$ and $r_{*}$ can be assigned. A further benefit of the presented approach is the possibility to gain a full understanding of the role of each density component in determining the resulting kinematical fields of the galaxy. For special density distributions, a further reduction of computational time is obtained by evaluating the potential with specific integral formulae, such as the Chandrasekhar formula for ellipsoidal distributions, and integrals involving Bessel functions for factorised disc distributions (see Appendix B).

In order to illustrate the features of our modeling procedure, we presented three galaxy models, composed of two stellar components, a spherical NFW DM halo and a central supermassive BH. In the JJE model, the total spherical stellar profile and one ellipsoidal stellar component, both Jaffe models, are assigned; the second stellar component is given by their difference. This model, when the DM halo is absent, has several properties available in analytical form (in particular in CMPZ21), and thus has been used to test the procedure and the code. The JHD model consists of a large and massive MN stellar disc, coupled with an ellipsoidal Jaffe stellar model, that can be seen as the stellar halo of a disc galaxy. In the JLD model, an ellipsoidal Jaffe component dominates in mass, and a MN stellar disc is small, inner and counter-rotating, as sometimes found in early-type galaxies.

As a fourth application, we explored the accuracy of one of the
3MN decompositions proposed by Smith et al. (2015) to reproduce the kinematical fields of double-exponential discs. We confirmed the excellent agreement of the rotation curves of the two models in the equatorial plane, at least out to $\sim 10 R_{d}$. We also found that $v_{\text {los }}$ tends to be larger for the double-exponential disc than for its $3 \mathrm{MN}$ representation, but overall the agreement is rather good, even outside the equatorial plane. A different situation is found for $\sigma_{\mathrm{los}}$ : the values are significantly larger in the $3 \mathrm{MN}$ model, which also presents a characteristic hourglass-shaped vertical distribution. Some care is thus recommended when using a $3 \mathrm{MN}$ decomposition to infer the properties of observed disc galaxies.

Ongoing applications of the presented modeling procedure, and in particular of JASMINE2, include the building of multi-component galaxy models for numerical simulations of gas flows in galaxies (e.g. Negri et al. 2014b; Gan et al. 2019a,b; Ciotti et al. in preparation); the study of circumnuclear stellar discs (also with counter-rotation, see e.g. Morelli et al. 2004; Krajnović et al. 2015; Mitzkus et al. 2017; Sormani et al. 2020; see also Cappellari 2016); a systematic exploration of galaxy models constrained to lie on the major observed Scaling Laws, extending the statistical approach pioneered in Bertin et al. (2002) and Lanzoni \& Ciotti (2003).

\section{ACKNOWLEDGEMENTS}

We are grateful to Antonio Mancino for independent checks of the results of JJE models. We thank the anonymous referee for useful comments that improved the paper. 

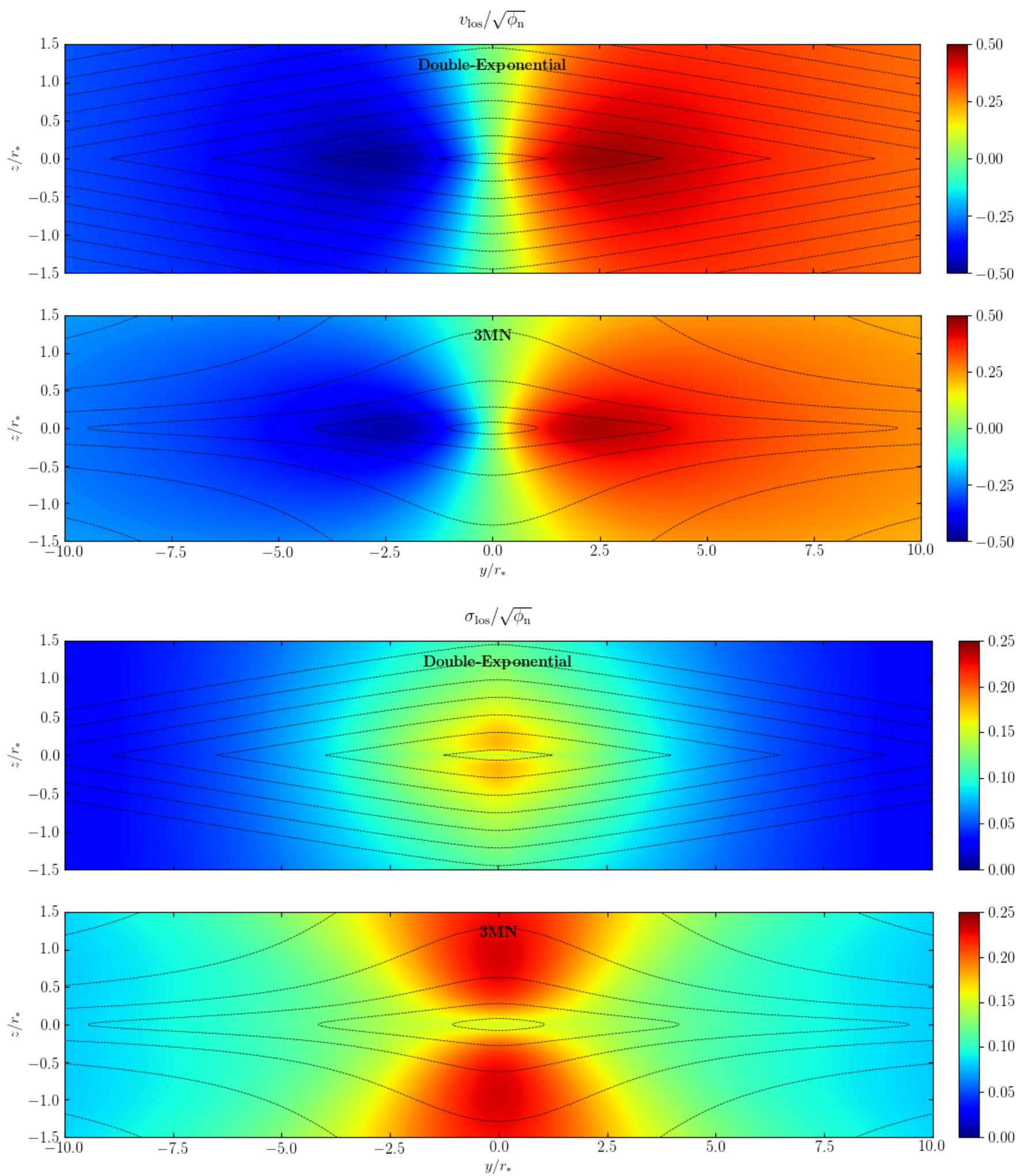

Figure 7. Edge-on projected rotational velocity $v_{\text {los }}$, and velocity dispersion $\sigma_{\text {los }}$, normalised to $\sqrt{\phi_{\mathrm{n}}}$, for the two models of Table 3 . The dotted contours are the same as in Figure 6. 


\section{DATA AVAILABILITY}

The data underlying this article were produced by the authors. They will be shared under reasonable request to the corresponding author.

\section{REFERENCES}

Bertin G., 2014, Dynamics of Galaxies. Cambridge University Press, doi:10.1017/CBO9780511731990

Bertin G., Ciotti L., Del Principe M., 2002, A\&A, 386, 149

Binney J., Tremaine S., 2008, Galactic Dynamics: Second Edition. Princeton University Press

Cappellari M., 2008, MNRAS, 390, 71

Cappellari M., 2016, ARAA, 54, 597

Caravita C., 2022, PhD Thesis. Bologna University

Ciotti L., 2021, Introduction to Stellar Dynamics. Cambridge University Press

Ciotti L., Bertin G., 2005, A\&A, 437, 419

Ciotti L., Pellegrini S., 1996, MNRAS, 279, 240

Ciotti L., Mancino A., Pellegrini S., Ziaee Lorzad A., 2021, MNRAS, 500, 1054

Ciotti L., Gan Z., Ostriker J. P., Pellegrini S., Caravita C., Mancino A., 2022, in preparation

Flynn C., Sommer-Larsen J., Christensen P. R., 1996, MNRAS, 281, 1027

Gan Z., Ciotti L., Ostriker J. P., Yuan F., 2019a, ApJ, 872, 167

Gan Z., Choi E., Ostriker J. P., Ciotti L., Pellegrini S., 2019b, ApJ, 875, 109

Jaffe W., 1983, MNRAS, 202, 995

Kormendy J., Ho L. C., 2013, ARA\&A, 51, 511

Krajnović D., et al., 2015, MNRAS, 452, 2

Kuzmin G. G., 1956, Azh, 33, 27

Lanzoni B., Ciotti L., 2003, A\&A, 404, 819

Maraston C., 2005, MNRAS, 362, 799

Mitzkus M., Cappellari M., Walcher C. J., 2017, MNRAS, 464, 4789

Miyamoto M., Nagai R., 1975, PASJ, 27, 533

Morelli L., et al., 2004, MNRAS, 354, 753

Navarro J. F., Frenk C. S., White S. D. M., 1996, ApJ, 462, 563

Negri A., Ciotti L., Pellegrini S., 2014a, MNRAS, 439, 823

Negri A., Posacki S., Pellegrini S., Ciotti L., 2014b, MNRAS, 445, 1351

Plummer H. C., 1911, MNRAS, 71, 460

Posacki S., Pellegrini S., Ciotti L., 2013, MNRAS, 433, 2259

Renzini A., Buzzoni A., 1986, in Chiosi C., Renzini A., eds, Astrophysics and Space Science Library Vol. 122, Spectral Evolution of Galaxies. pp 195-231, doi:10.1007/978-94-009-4598-2_19

Rojas-Niño A., Read J. I., Aguilar L., Delorme M., 2016, MNRAS, 459, 3349

Satoh C., 1980, PASJ, 32, 41

Smet C. O., Posacki S., Ciotti L., 2015, MNRAS, 448, 2921

Smith R., Flynn C., Candlish G. N., Fellhauer M., Gibson B. K., 2015, MNRAS, 448, 2934

Sormani M. C., Magorrian J., Nogueras-Lara F., Neumayer N., Schönrich R., Klessen R. S., Mastrobuono-Battisti A., 2020, MNRAS, 499, 7

Yoon D., Yuan F., Ostriker J. P., Ciotti L., Zhu B., 2019, ApJ, 885, 16

\section{APPENDIX A: POSITIVITY CONDITION FOR JJE MODELS}

The stellar component $\rho_{* 2}$, for the 2-component ellipsoidal models described in Section 4.1, is given by the difference of an assigned total $\rho_{*}$ and an assigned $\rho_{* 1}$. This approach naturally leads to discuss the positivity of $\rho_{* 2}$, with a treatment similar to that followed in the appendix of CMPZ21, and references therein. We generalise equation (46) as

$\frac{\rho_{* 2}(R, z)}{(3-\gamma) \rho_{\mathrm{n}}}=\frac{\xi}{q m^{\gamma}(\xi+m)^{4-\gamma}}-\frac{\mathcal{R}_{1} \xi_{1}}{q_{1} m_{1}^{\gamma}\left(\xi_{1}+m_{1}\right)^{4-\gamma}}$,

recovering the case of JJE models for $\gamma=2$. In order to discuss the positivity condition for $\rho_{* 2}$, we use spherical coordinates, so that
$(R, z)=r(\sin \theta, \cos \theta)$ and

$m=s \Omega, \quad m_{1}=s \Omega_{1}, \quad s \equiv \frac{r}{r_{*}}$,

where

$\Omega^{2} \equiv \sin ^{2} \theta+\frac{\cos ^{2} \theta}{q^{2}}, \quad \Omega_{1}^{2} \equiv \sin ^{2} \theta+\frac{\cos ^{2} \theta}{q_{1}^{2}}$.

The positivity of $\rho_{* 2}$ reduces to a condition on $\mathcal{R}_{1}$, given by

$\mathcal{R}_{1} \leq \mathcal{R}_{\mathrm{M}} \equiv \inf _{\mathcal{I}}\left[\frac{\xi q_{1}}{\xi_{1} q}\left(\frac{\Omega_{1}}{\Omega}\right)^{\gamma}\left(\frac{\xi_{1}+s \Omega_{1}}{\xi+s \Omega}\right)^{4-\gamma}\right]$,

over the rectangular region $\mathcal{I} \equiv\{s \geq 0,0 \leq \theta \leq \pi / 2\}$ in the $(s, \theta)$ plane. Following the discussion in CMPZ21, we determine

$\mathcal{R}_{\mathrm{M}}=\min \left(\mathcal{R}_{\mathrm{c}}, \mathcal{R}_{\infty}, \mathcal{R}_{0}, \mathcal{R}_{\pi / 2}, \mathcal{R}_{\text {int }}\right)$,

where the first four quantities refer to the minimum value of the r.h.s. of equation (A4) over the boundaries of $\mathcal{I}$, and $\mathcal{R}_{\text {int }}$ is the value of a minimum (if it exists) in the interior of $\mathcal{I}$. When $q_{1} \neq q$, it is simple to show that no critical points can exist in the interior of $\mathcal{I}$, and so the discussion reduces to the boundaries of $\mathcal{I}$ : geometrically, $\mathcal{R}_{\mathrm{M}}$ can be reached only at the centre $\left(s=0, \mathcal{R}_{\mathrm{c}}\right)$, at infinity $\left(s \rightarrow \infty, \mathcal{R}_{\infty}\right)$, along the symmetry axis $\left(\theta=0, \mathcal{R}_{0}\right)$, or on the equatorial plane $(\theta=\pi / 2$, $\left.\mathcal{R}_{\pi / 2}\right)$.

We begin with $\mathcal{R}_{\mathrm{c}}$ and $\mathcal{R}_{\infty}$, obtaining

$\mathcal{R}_{\mathrm{c}}=\frac{\xi_{1}^{3-\gamma} q_{1}}{\xi^{3-\gamma} q} \min _{0 \leq \theta \leq \pi / 2}\left(\frac{\Omega_{1}}{\Omega}\right)^{\gamma}$,

$\mathcal{R}_{\infty}=\frac{\xi q_{1}}{\xi_{1} q} \min _{0 \leq \theta \leq \pi / 2}\left(\frac{\Omega_{1}}{\Omega}\right)^{4}$.

Now, from equation (A3), it is easy to show that for a generic $\alpha \geq 0$, the function $\left(\Omega_{1} / \Omega\right)^{\alpha}$ reaches its minimum at $\theta=\pi / 2$ if $q_{1} \leq q$, and at $\theta=0$ if $q \leq q_{1}$, so that

$\min _{0 \leq \theta \leq \pi / 2}\left(\frac{\Omega_{1}}{\Omega}\right)^{\alpha}=\left\{\begin{array}{l}1, \quad q_{1} \leq q, \\ \left(\frac{q}{q_{1}}\right)^{\alpha}, \quad q \leq q_{1},\end{array}\right.$

and the conditions in equations (A6) and (A7) can be finally summarised as

$\mathcal{R}_{\mathrm{c}}=\frac{\xi_{1}^{3-\gamma} q_{1}}{\xi^{3-\gamma} q} \min \left(1, \frac{q^{\gamma}}{q_{1}^{\gamma}}\right)$

$\mathcal{R}_{\infty}=\frac{\xi q_{1}}{\xi_{1} q} \min \left(1, \frac{q^{4}}{q_{1}^{4}}\right)$.

Along the symmetry axis, and in the equatorial plane, condition (A4) becomes

$\mathcal{R}_{0}=\frac{\xi q^{3}}{\xi_{1} q_{1}^{3}} \inf _{0 \leq s<\infty}\left(\frac{\xi_{1} q_{1}+s}{\xi q+s}\right)^{4-\gamma}$,

$\mathcal{R}_{\pi / 2}=\frac{\xi q_{1}}{\xi_{1} q} \inf _{0 \leq s<\infty}\left(\frac{\xi_{1}+s}{\xi+s}\right)^{4-\gamma}$,

and simple algebra finally shows that the results can be summarised as

$\mathcal{R}_{0}=\frac{\xi q^{3}}{\xi_{1} q_{1}^{3}} \min \left[1,\left(\frac{\xi_{1} q_{1}}{\xi q}\right)^{4-\gamma}\right]$, 
$\mathcal{R}_{\pi / 2}=\frac{\xi q_{1}}{\xi_{1} q} \min \left(1, \frac{\xi_{1}^{4-\gamma}}{\xi^{4-\gamma}}\right)$.

For the JJE models in Section 4.1, with $\xi_{1}<\xi, q_{1}<q$, and $\gamma=2$, the positivity condition (A5) becomes

$\mathcal{R}_{1} \leq \mathcal{R}_{\mathrm{M}}=\frac{\xi_{1} q_{1}}{\xi q}$

\section{APPENDIX B: POTENTIAL OF FACTORISED EXPONENTIAL DISCS}

Due to the importance in applications, here we summarise the main results about the numerical evaluation of the potential produced by factorised exponential discs as in equation (51), by using the technique of Bessel functions. From equations (2.103) and (2.114) in Ciotti (2021), the potential can be easily written in full generality as

$\phi(R, z)=-2 \pi G \rho_{0} r_{*}^{2} \alpha^{2} \beta \int_{0}^{\infty} \frac{\mathrm{J}_{0}(\lambda \tilde{R}) \hat{V}(\lambda \beta, \tilde{z} / \beta)}{\left(1+\alpha^{2} \lambda^{2}\right)^{3 / 2}} \mathrm{~d} \lambda$,

where $\mathrm{J}_{0}$ is a Bessel function of the first kind, $\alpha=R_{\mathrm{d}} / r_{*}, \beta=h / r_{*}$, $\tilde{R}=R / r_{*}, \tilde{z}=z / r_{*}$, and finally

$\hat{V}(\gamma, x) \equiv \int_{-\infty}^{\infty} \mathrm{e}^{-\gamma|| x|-t|} V(|t|) \mathrm{d} t$.

Therefore, once the function $\hat{V}$ is known analytically, the integration for each grid point $(\tilde{R}, \tilde{z})$ reduces to a fast 1-dimensional integration, instead of the more time-consuming 2-dimensional integration that would be required when using the standard formula based on complete elliptic integrals (equation 42), or the alternative equation based on modified Bessel functions (equation 2.170 in BT08; equation 4 in Smith et al. 2015).

For the three discs considered in Smith et al. (2015), we define $\mathcal{R}_{\mathrm{d}}=M_{\mathrm{d}} / M_{*}$, where $M_{\mathrm{d}}$ is the total mass of the disc, so that for the double-exponential disc considered in Section 4.3.1, we have

$\rho_{*}(R, z)=\frac{\rho_{\mathrm{n}} \mathcal{R}_{\mathrm{d}}}{\alpha^{2} \beta} \mathrm{e}^{-\tilde{R} / \alpha-|\tilde{z}| / \beta}, \quad \hat{V}(\gamma, x)=\frac{2\left(\gamma \mathrm{e}^{-|x|}-\mathrm{e}^{-\gamma|x|}\right)}{\gamma^{2}-1}$,

for $\gamma \neq 1$, and $\hat{V}(1, x)=(1+|x|) \mathrm{e}^{-|x|}$. For completness, we also report the formulae for the razor-thin exponential disc,

$\rho_{*}(R, z)=\frac{2 \rho_{\mathrm{n}} \mathcal{R}_{\mathrm{d}}}{\alpha^{2} \beta} \mathrm{e}^{-\tilde{R} / \alpha} \delta(\tilde{z} / \beta), \quad \hat{V}(\gamma, x)=\mathrm{e}^{-\gamma|x|}$,

where $\delta$ is the Dirac- $\delta$ function, and for the "pseudo-isothermal" exponential disc,

$\rho_{*}(R, z)=\frac{2^{2-a} \rho_{\mathrm{n}} \mathcal{R}_{\mathrm{d}}}{\alpha^{2} \beta \mathrm{B}(a / 2, a / 2)} \frac{\mathrm{e}^{-\tilde{R} / \alpha}}{\cosh (\tilde{z} / \beta)^{a}}, \quad a>0$,

where $\mathrm{B}(x, y)$ is the Euler complete Beta function, and

$$
\begin{aligned}
\hat{V}(\gamma, x)= & 2^{a-1} \mathrm{e}^{-\gamma|x|} \mathrm{B}\left(\frac{a+\gamma}{2}, \frac{a-\gamma}{2}, \frac{\mathrm{e}^{2|x|}}{1+\mathrm{e}^{2|x|}}\right)+ \\
& 2^{a-1} \mathrm{e}^{\gamma|x|} \mathrm{B}\left(\frac{a+\gamma}{2}, \frac{a-\gamma}{2}, \frac{1}{1+\mathrm{e}^{2|x|}}\right) .
\end{aligned}
$$

For computational reasons, it can be convenient to express the incomplete Beta functions above by using their hypergeometric expression

$\mathrm{B}(a, b ; z)=\frac{z^{a}}{a}{ }_{2} F_{1}(a, 1-b, 1+a ; z)$.
We verified the numerical accuracy of the 1-dimensional integration of equations (B1)-(B3) by comparison with the potential obtained from equation (42).

We conclude by noticing that equation (B1) can be immediately extended to other families of factorised thick discs, with a radial density factor allowing for an explicit Hankel transform. Examples of these thick discs (implemented in JASMINE2), are the Kuzmin disc, the truncated, untruncated and finite Mestel discs, the truncated constant density disc, and the Maclaurin disc (for the relative Hankel transform, see respectively equations 13.148, 2.119, 5.46, 5.55, 5.53, 5.54 in Ciotti 2021; see also Caravita in preparation).

This paper has been typeset from a $\mathrm{T}_{\mathrm{E}} \mathrm{X} / \mathrm{L} \mathrm{T}_{\mathrm{E}} \mathrm{X}$ file prepared by the author. 